\title{
REGULARITY OF BICYCLIC GRAPHS AND THEIR POWERS
}

\author{
YAIRON CID-RUIZ, SEPEHR JAFARI, NAVID NEMATI, AND BEATRICE PICONE
}

\begin{abstract}
Let $I(G)$ be the edge ideal of a bicyclic graph $G$ with a dumbbell as the base graph. In this paper, we characterize the Castelnuovo-Mumford regularity of $I(G)$ in terms of the induced matching number of $G$. For the base case of this family of graphs, i.e. dumbbell graphs, we explicitly compute the induced matching number. Moreover, we prove that $\operatorname{reg} I(G)^{q}=2 q+\operatorname{reg} I(G)-2$, for all $q \geq 1$, when $G$ is a dumbbell graph with a connecting path having no more than two vertices.
\end{abstract}

\section{INTRODUCTION}

Let $I$ be a homogeneous ideal in a polynomial ring $R=\mathbb{k}\left[x_{1}, \ldots, x_{r}\right]$ over a field $\mathbb{k}$. The Castelnuovo-Mumford regularity of $I$, denoted by reg $(I)$, has been an interesting and active research topic for the past decades. There exists a vast literature on the study of reg $(I)$ (see e.g. [9]). A celebrated result on the behavior of the regularity of powers of ideals was given independently by Cutkosky, Herzog, and Trung in [10], and by Kodiyalam in [24]. In both papers, it is proved that for all $q \geq q_{0}$, the regularity of the powers of $I$ is asymptotically a linear function $\operatorname{reg}\left(I^{q}\right)=d q+b$, where $q_{0}$ is the so-called stabilizing index, and $b$ is the so-called constant. The value of $d$ in the above formula is well understood (see [28, Theorem 3.2]). For example, $d$ is equal to the degree of the generators of $I$ when $I$ is equigenerated in degree $d$ (see loc. cit.). However, there is no general or precise method to determine $q_{0}$ and $b$.

In recent years, many researchers have tried to compute $q_{0}$ and $b$ for special families of ideals. The most simple case, yet interesting, is when $I$ is the edge ideal of a finite simple graph. Let $G=(V(G), E(G))$ denote a finite simple undirected graph. Let $R$ be the polynomial ring $\mathbb{k}\left[x_{i} \mid x_{i} \in V(G)\right]$ where $\mathbb{k}$ is any field. The edge ideal $I(G)$ of $G$ is the ideal

$$
I(G)=\left(x_{i} x_{j} \mid\left\{x_{i}, x_{j}\right\} \in E(G)\right) .
$$

Several authors have settled the problem of determining the stabilizing index and the constant for special families of graphs. Banerjee proved that $\operatorname{reg} I(G)^{q}=2 q$, for all $q \geq 2$, when $G$ is a gap-free and cricket-free graph (see [4]). Beyarslan, Hà, and Trung settled the problem for the family of forests and cycles (see [6]). Moghimian, Fakhari, and Yassemi answered the question for the family of whiskered cycles (see [26]). Their results were expanded to the family of unicyclic graphs by Alilooee, Beyarslan, and Selvaraja (see [2]). Moreover, Alilooee and Banerjee determined the stabilizing index and the constant for the family of bipartite graphs with regularity equal to three (see [1]). Jayanthan and Selvaraja settled the problem for the family of very well-covered graphs (see [21]). Recently, Erey proved that if $G$ is a gap-free and diamond-free graph, then $\operatorname{reg} I(G)^{q}=2 q$ for all $q \geq 2$ (see [13]). The approach is focused on the relations between the combinatorics of graphs

2010 Mathematics Subject Classification. 13D02, 05C25, 05C38, 05E40.

Key words and phrases. bicyclic graphs, edge ideals, regularity, induced matching number, Lozin transformation, even-connection.

The first named author was funded by the European Union's Horizon 2020 research and innovation programme under the Marie Skłodowska-Curie grant agreement No. 675789. 
and algebraic properties of edge ideals. We refer the reader to see [5], [23], [17], [8], [20], [3], [29] and [27] for more information on this topic. The main purpose of this paper is to extend the results of [2] to the family of bicyclic graphs (i.e. a graph with two cycles).

The family of bicyclic graphs has three possible types of base graphs: two cycles joined at a vertex, two cycles connected by a path and two cycles sharing a path. In [15], Gu computed the regularity of all the powers of the edge ideal of a base graph of the the first type (i.e. two cycles joined at a vertex).

In this paper, we consider the family of bicyclic graphs where the base graph is a dumbbell. A dumbbell graph $C_{n} \cdot P_{l} \cdot C_{m}$ is a graph consisting of two cycles $C_{n}$ and $C_{m}$ connected with a path $P_{l}$, where $n, m$, and $l$ are the number of vertices (see Example 2.1). For convenience of notation, we define the following function

$$
\xi_{3}(n)= \begin{cases}1 & \text { if } n \equiv 0,1(\bmod 3), \\ 0 & \text { if } n \equiv 2(\bmod 3) .\end{cases}
$$

Here, we describe the basic outline and main results of this paper.

In Section 1, we fix some notations and recall known results which are important to our approach.

In Section 2, we use combinatorial techniques to compute the induced matching number of a dumbbell graph. Then, applying inductive methods, we study the regularity of the edge ideals of dumbbell graphs. For a dumbbell graph $C_{n} \cdot P_{l} \cdot C_{m}$, we assume that " $n \bmod 3 \leq m \bmod 3$ ". Since the graphs $C_{n} \cdot P_{l} \cdot C_{m}$ and $C_{m} \cdot P_{l} \cdot C_{n}$ are clearly isomorphic, the cases " $n \equiv 2(\bmod 3), m \equiv 0,1(\bmod 3)$ " have the same results as the cases " $n \equiv 0,1(\bmod 3), m \equiv 2(\bmod 3)$ ". Our approach is based on the Lozin transformation (see [25] and [7]), and the induced matching number of a dumbbell graph. The following results are given in this section:

Theorem A (Theorem 2.4). Let $n, m \geq 3$ and $l \geq 1$, then

$$
\nu\left(C_{n} \cdot P_{l} \cdot C_{m}\right)=\left\lfloor\frac{n}{3}\right\rfloor+\left\lfloor\frac{m}{3}\right\rfloor+\left\lfloor\frac{l-\xi_{3}(n)-\xi_{3}(m)+1}{3}\right\rfloor .
$$

Theorem B (Theorem 2.16). Let $m, n \geq 3$ and $l \geq 1$,

(i) if $l \equiv 0,1(\bmod 3)$, then

$$
\operatorname{reg} I\left(C_{n} \cdot P_{l} \cdot C_{m}\right)= \begin{cases}\nu\left(C_{n} \cdot P_{l} \cdot C_{m}\right)+2 & \text { if } n, m \equiv 2(\bmod 3), \\ \nu\left(C_{n} \cdot P_{l} \cdot C_{m}\right)+1 & \text { otherwise; }\end{cases}
$$

(ii) if $l \equiv 2(\bmod 3)$, then

$$
\operatorname{reg} I\left(C_{n} \cdot P_{l} \cdot C_{m}\right)= \begin{cases}\nu\left(C_{n} \cdot P_{l} \cdot C_{m}\right)+2 & \text { if } n \equiv 0,1(\bmod 3), m \equiv 2(\bmod 3) \\ \nu\left(C_{n} \cdot P_{l} \cdot C_{m}\right)+1 & \text { otherwise }\end{cases}
$$

In Section 3, for a bicyclic graph $G$ having a dumbbell graph as the base, we give a combinatorial characterization of $\operatorname{reg} I(G)$ in terms of the induced matching number $\nu(G)$.

Theorem $\mathbf{C}$ (Theorem 3.24). Let $G$ be a bicyclic graph with dumbbell $C_{n} \cdot P_{l} \cdot C_{m}$. The following statements hold.

(I) Let $n, m \equiv 0,1(\bmod 3)$, then $\operatorname{reg} I(G)=\nu(G)+1$.

(II) Let $n \equiv 0,1(\bmod 3)$ and $m \equiv 2(\bmod 3)$, then

$$
\nu(G)+1 \leq \operatorname{reg} I(G) \leq \nu(G)+2,
$$

and $\operatorname{reg} I(G)=\nu(G)+2$ if and only if $\nu(G)=\nu\left(G \backslash \Gamma_{G}\left(C_{m}\right)\right)$. 
(III) Let $n, m \equiv 2(\bmod 3)$ and $l \geq 3$, then $\nu(G)+1 \leq \operatorname{reg} I(G) \leq \nu(G)+3$. Moreover:

(i) $\operatorname{reg} I(G)=\nu(G)+3$ if and only if $\nu\left(G \backslash \Gamma_{G}\left(C_{n} \cup C_{m}\right)\right)=\nu(G)$.

(ii) $\operatorname{reg} I(G)=\nu(G)+1$ if and only if the following conditions hold:

(a) $\nu(G)-\nu\left(G \backslash \Gamma_{G}\left(C_{n} \cup C_{m}\right)\right)>1$;

(b) $\nu(G)>\nu\left(G \backslash \Gamma_{G}\left(C_{n}\right)\right)$;

(c) $\nu(G)>\nu\left(G \backslash \Gamma_{G}\left(C_{m}\right)\right)$.

(IV) Let $n, m \equiv 2(\bmod 3)$ and $l \leq 2$, then $\nu(G)+1 \leq \operatorname{reg} I(G) \leq \nu(G)+2$. If $x$ is a vertex on $P_{l}$ and $\mathcal{L}_{x}(G)$ is the Lozin transformation of $G$ with respect to $x$, then $\operatorname{reg} I(G)=\nu(G)+1$ if and only if the following conditions are satisfied:
(a) $\nu\left(\mathcal{L}_{x}(G)\right)-\nu\left(\mathcal{L}_{x}(G) \backslash \Gamma_{\mathcal{L}_{x}(G)}\left(C_{n} \cup C_{m}\right)\right)>1$;
(b) $\nu\left(\mathcal{L}_{x}(G)\right)>\nu\left(\mathcal{L}_{x}(G) \backslash \Gamma_{\mathcal{L}_{x}(G)}\left(C_{n}\right)\right)$;
(c) $\nu\left(\mathcal{L}_{x}(G)\right)>\nu\left(\mathcal{L}_{x}(G) \backslash \Gamma_{\mathcal{L}_{x}(G)}\left(C_{m}\right)\right)$.

In Section 4, we investigate the asymptotic behavior of regularity of powers of $I\left(C_{n} \cdot P_{l} \cdot C_{m}\right)$ when $l \leq 2$. The approach takes advantage of the notion of even-connectedness and the relations between the induced matching number of graphs and the regularity of the edge ideal.

Theorem D (Theorem 4.7). Let $C_{n} \cdot P_{l} \cdot C_{m}$ with $l \leq 2$, then

$$
\operatorname{reg} I\left(C_{n} \cdot P_{l} \cdot C_{m}\right)^{q}=2 q+\operatorname{reg} I\left(C_{n} \cdot P_{l} \cdot C_{m}\right)-2
$$

for any $q \geq 1$.

The above equality is no longer true for the case $l \geq 3$ as there are immediate counter examples (see Remark 4.9).

\section{Preliminaries}

Let $R=\mathbb{k}\left[x_{1}, \ldots, x_{r}\right]$ be a standard graded polynomial ring over a field $\mathbb{k}$ and let $\mathfrak{m}=\left(x_{1}, \ldots, x_{r}\right)$ be its maximal irrelevant ideal. For a graded $R$-module $M$, one can define the Castelnuovo-Mumford regularity in different ways. We recall the definition of the regularity of an $R$-module $M$ given in terms of the minimal free resolution of $M$. The minimal graded free resolution of $M$ is an exact sequence of the form

$$
0 \rightarrow F_{p} \rightarrow F_{p-1} \rightarrow \cdots \rightarrow F_{0} \rightarrow M \rightarrow 0,
$$

where each $F_{i}$ is a graded free $R$-module of the form $F_{i}=\bigoplus_{j \in \mathbb{N}} R(-j)^{\beta_{i, j}(M)}$, each $\varphi_{i}: F_{i} \rightarrow$ $F_{i-1}$, with $F_{-1}:=M$, is a graded homomorphism of degree zero such that $\varphi_{i+1}\left(F_{i+1}\right) \subseteq \mathfrak{m} F_{i}$ for all $i \geq 0$. The number $\beta_{i, j}(M)$, called the $(i, j)^{\text {th }}$-graded Betti number of $M$, is an important invariant of the module $M$. In particular, the number $\beta_{i}(M)=\sum_{j \in \mathbb{N}} \beta_{i, j}(M)$ is called the $i$-th Betti number of $M$. Note that the minimal free resolution of $M$ is unique up to isomorphism, hence the graded Betti numbers are uniquely determined.

Definition 1.1. Let $M$ be a finitely generated graded $R$-module. The regularity of $M$ is given by

$$
\operatorname{reg}(M)=\max \left\{j-i \mid \beta_{i, j}(M) \neq 0\right\} .
$$

Remark 1.2. Note that, if $I$ is a graded ideal of $R$, then $\operatorname{reg}(R / I)=\operatorname{reg}(I)-1$.

Let $G=(V, E)$ be a graph with vertex set $V=\left\{x_{1}, \ldots, x_{r}\right\}$. Here, we recall some classes of graphs that we need for this study.

Definition 1.3. Let $G=(V, E)$ be a graph. 
(i) $G$ is called a path on $l$ vertices, denoted by $P_{l}$, if $V=\left\{x_{1}, \ldots, x_{l}\right\}$ and $\left\{x_{i}, x_{i+1}\right\} \in E$ for all $1 \leq i \leq l-1$.

(ii) $G$ is called a cycle on $n$ vertices, denoted by $C_{n}$, if $V=\left\{x_{1}, \ldots, x_{n}\right\}$ and $\left\{x_{i}, x_{i+1}\right\} \in$ $E$ for all $1 \leq i \leq n-1$ and $\left\{x_{n}, x_{1}\right\} \in E$.

(iii) $G$ is called a dumbbell graph if $G$ contains two cycles $C_{n}$ and $C_{m}$ joined by a path $P_{l}$ on $l$ vertices. We denote it by $C_{n} \cdot P_{l} \cdot C_{m}$ (see Example 2.1).

For a vertex $u$ in a graph $G=(V, E)$, let $N_{G}(u)=\{v \in V \mid\{u, v\} \in E\}$ be the set of neighbors of $u$, and set $N_{G}[u]:=N_{G}(u) \cup\{u\}$. An edge $e$ is incident to a vertex $u$ if $u \in e$. The degree of a vertex $u \in V$, denoted by $\operatorname{deg}_{G}(u)$, is the number of edges incident to $u$. When there is no confusion, we omit $G$ and write $N(u), N[u]$ and $\operatorname{deg}(u)$. For an edge $e$ in a graph $G=(V, E)$, we define $G \backslash e$ to be the subgraph of $G$ obtained by deleting $e$ from $E$ (but the vertices are maintained).

Let $G=(V, E)$ be a graph and $W \subseteq V$, the induced subgraph of $G$ on $W$, denoted by $G[W]$, is the graph with vertex set $W$ and edge set $\{e \in E \mid e \subseteq W\}$. For a subset $W \subseteq V$ of the vertices in $G$, we define $G \backslash W$ to be the induced subgraph of $G$ obtained by deleting the vertices of $W$ and their incident edges from $G$. When $W=\{u\}$ consists of a single vertex, we write $G \backslash u$ instead of $G \backslash\{u\}$. For an edge $e=\{u, v\} \in E$, let $N_{G}[e]=N_{G}[u] \cup N_{G}[v]$ and define $G_{e}$ to be the induced subgraph of $G$ over the vertex set $V \backslash N_{G}[e]$.

One can think of the vertices of $G=(V, E)$ as the variables of the polynomial ring $R=\mathbb{k}\left[x_{1}, \ldots, x_{r}\right]$ for convenience. Similarly, the edges of $G$ can be considered as square free monomials of degree two. By an abuse of notation, we use $e$ to refer to both the edge $e=\left\{x_{i}, x_{j}\right\} \in E$ and the monomial $e=x_{i} x_{j} \in I(G)$.

Definition 1.4. Let $G=(V, E)$ be a graph.

$A$ collection $C$ of edges of $G$ is called a matching if the edges in $C$ are pairwise disjoint. The maximum size of a matching in $G$ is called its matching number, which is denoted by $\operatorname{match}(G)$.

$A$ collection $C$ of edges of $G$ is called an induced matching if $C$ is a matching, and $C$ consists of all edges of the induced subgraph $G\left[\bigcup_{e \in C} e\right]$ of $G$. The maximum size of an induced matching in $G$ is called its induced matching number and it is denoted by $\nu(G)$.

Remark 1.5. [6, Remark 2.12] Let $P_{l}$ be a path on l vertices, then we have

$$
\nu\left(P_{l}\right)=\left\lfloor\frac{l+1}{3}\right\rfloor
$$

Remark 1.6. [6, Remark 2.13] Let $C_{n}$ be a cycle on $n$ vertices, then we have

$$
\nu\left(C_{n}\right)=\left\lfloor\frac{n}{3}\right\rfloor .
$$

Depending on $r=n \bmod 3$ we can assume the following:

(i) when $r=0$, there exists a maximal induced matching of $C_{n}$ that does not contain the edges $x_{1} x_{2}$ and $x_{1} x_{n}$;

(ii) when $r=1$, there exists a maximal induced matching of $C_{n}$ that does not contain the edges $x_{1} x_{2}, x_{1} x_{n}$ and $x_{n-1} x_{n}$;

(iii) when $r=2$, there exists a maximal induced matching of $C_{n}$ that does not contain the edges $x_{1} x_{2}, x_{2} x_{3}, x_{1} x_{n}$ and $x_{n-1} x_{n}$.

Theorem 1.7. [16, Lemma 3.1, Theorems 3.4 and 3.5] Let $G=(V, E)$ be a graph.

(i) If $H$ is an induced subgraph of $G$, then $\operatorname{reg} I(H) \leq \operatorname{reg} I(G)$; 
(ii) Let $x \in V$, then

$$
\operatorname{reg} I(G) \leq \max \{\operatorname{reg} I(G \backslash x), \operatorname{reg} I(G \backslash N[x])+1\} ;
$$

(iii) Let $e \in E$, then

$$
\operatorname{reg} I(G) \leq \max \left\{2, \operatorname{reg} I(G \backslash e), \operatorname{reg} I\left(G_{e}\right)+1\right\} .
$$

Now we recall the concept of even-connection introduced by Banerjee in [4].

Definition $1.8([4])$. Let $G=(V, E)$ be a graph with edge ideal $I=I(G)$. Two vertices $x_{i}$ and $x_{j}$ in $G$ are called even-connected with respect to an $s$-fold product $M=e_{1} \cdots e_{s}$, where $e_{1}, \ldots, e_{s}$ are edges in $G$, if there is a path $p_{0}, \ldots, p_{2 l+1}$, for some $l \geq 1$, in $G$ such that the following conditions hold:

(i) $p_{0}=x_{i}$ and $p_{2 l+1}=x_{j}$;

(ii) for all $0 \leq j \leq l-1,\left\{p_{2 j+1}, p_{2 j+2}\right\}=e_{i}$ for some $i$;

(iii) for all $i,\left|\left\{j \mid\left\{p_{2 j+1}, p_{2 j+2}\right\}=e_{i}\right\}\right| \leq\left|\left\{t \mid e_{t}=e_{i}\right\}\right|$.

Theorem 1.9. [4, Theorems 6.1 and 6.5] Let $M=e_{1} e_{2} \cdots e_{s}$ be a minimal generator of $I^{s}$. Then $\left(I^{s+1}: M\right)$ is minimally generated by monomials of degree 2 , and uv ( $u$ and $v$ may be the same) is a minimal generator of $\left(I^{s+1}: M\right)$ if and only if either $\{u, v\} \in E$ or $u$ and $v$ are even-connected with respect to $M$.

Remark 1.10. [4, Lemma 6.11] Let $\left(I^{s+1}: M\right)^{\text {pol }}$ be the polarization of the ideal $\left(I^{s+1}: M\right)$ (see e.g. $[19, \S 1.6])$. From the previous theorem we can construct a graph $G^{\prime}$ whose edge ideal is given by $\left(I^{s+1}: M\right)^{\text {pol }}$. The new graph $G^{\prime}$ is given by:

(i) All the vertices and edges of $G$.

(ii) Any two vertices $u, v, u \neq v$ that are even-connected with respect to $M$ are connected by an edge in $G^{\prime}$.

(iii) For every vertex $u$ which is even-connected to itself with respect to $M$, there is a new vertex $u^{\prime}$ which is connected to $u$ by an edge and not connected to any other vertex (so $\mathrm{uu}^{\prime}$ is a whisker).

Theorem 1.11. [4, Theorem 5.2] Let $G$ be a graph and $\left\{m_{1}, \ldots, m_{r}\right\}$ be the set of minimal monomial generators of $I(G)^{q}$ for all $q \geq 1$, then

$$
\operatorname{reg} I(G)^{q+1} \leq \max \left\{\operatorname{reg}\left(I(G)^{q}: m_{l}\right)+2 q, 1 \leq l \leq r, \operatorname{reg} I(G)^{q}\right\} .
$$

We recall a result by Kalai and Meshulam on the regularity of monomial ideals.

Theorem 1.12. ([22], [18]) Let $I_{1}, \ldots, I_{s}$ be monomial ideals in $R$, then

$$
\operatorname{reg}\left(R / \sum_{i=1}^{s} I_{i}\right) \leq \sum_{i=1}^{s} \operatorname{reg}\left(R / I_{i}\right) .
$$

In the particular case of edge ideals we have the following upper bound.

Corollary 1.13. Let $G$ be a simple graph. If $G_{1}, \ldots, G_{s}$ are subgraphs of $G$ such that $E(G)=\bigcup_{i=1}^{s} E\left(G_{i}\right)$ then

$$
\operatorname{reg}(R / I(G)) \leq \sum_{i=1}^{s} \operatorname{reg}\left(R / I\left(G_{i}\right)\right) .
$$

The previous upper bound is sharp when $G$ is a disjoint union of the graphs $G_{1}, \ldots, G_{s}$. 
Corollary 1.14. [5, Corollary 3.10] Let $G$ be a simple graph. If $G$ can be written as a disjoint union of graphs $G_{1}, \ldots, G_{s}$ then

$$
\operatorname{reg}(R / I(G))=\sum_{i=1}^{s} \operatorname{reg}\left(R / I\left(G_{i}\right)\right)
$$

The regularity of the edge ideal of a forest was first computed by Zheng in [30, Theorem $2.18]$.

Theorem 1.15. [30, Theorem 2.18] Let $G$ be a forest, then

$$
\operatorname{reg} I(G)=\nu(G)+1 .
$$

In [23] Katzman proved that the previous equality is a lower bound for any graph.

Theorem 1.16. [23, Corollary 1.2] Let $G$ be a graph, then

$$
\operatorname{reg} I(G) \geq \nu(G)+1 \text {. }
$$

The decycling number of a graph is an important combinatorial invariant which can be used to obtain an upper bound for the regularity of the edge ideal of a graph.

Definition 1.17. For a graph $G$ and $D \subset V(G)$, if $G \backslash D$ is acyclic, i.e. contains no induced cycle, then $D$ is said to be a decycling set of $G$. The size of a smallest decycling set of $G$ is called the decycling number of $G$ and denoted by $\nabla(G)$.

Theorem 1.18. [7, Theorem 4.11] Let $G$ be a graph, then

$$
\operatorname{reg} I(G) \leq \nu(G)+\nabla(G)+1 .
$$

In [6] Beyarslan, Hà and Trung provided a formula for the regularity of the powers of edge ideals of forests and cycles in terms of the induced matching number.

Theorem 1.19. [6, Theorem 4.7] Let $G$ be a forest, then

$$
\operatorname{reg} I(G)^{q}=2 q+\nu(G)-1 .
$$

for all $q \geq 1$.

Theorem 1.20. [6, Theorem 5.2]. Let $C_{n}$ be a cycle with $n$ vertices, then

$$
\operatorname{reg} I\left(C_{n}\right)= \begin{cases}\nu\left(C_{n}\right)+1 & \text { if } n \equiv 0,1(\bmod 3), \\ \nu\left(C_{n}\right)+2 & \text { if } n \equiv 2(\bmod 3),\end{cases}
$$

where $\nu\left(C_{n}\right)=\left\lfloor\frac{n}{3}\right\rfloor$ denote the induced matching number of $C_{n}$. Moreover,

$$
\operatorname{reg} I\left(C_{n}\right)^{q}=2 q+\nu\left(C_{n}\right)-1 .
$$

and for all $q \geq 2$.

In addition, the authors of [6] gave a lower bound for the regularity of the powers of the edge ideal of an arbitrary graph, and an upper bound for the regularity of the edge ideal of a graph containing a Hamiltonian path.

Theorem 1.21. [6, Theorem 4.5] Let $G$ be a graph and let $\nu(G)$ denote its induced matching number. Then, for all $q \geq 1$, we have

$$
\operatorname{reg} I(G)^{q} \geq 2 q+\nu(G)-1 .
$$

Theorem 1.22. [6, Theorem 3.1] Let $G$ be a graph on $n$ vertices. If $G$ contains a Hamiltonian path, then

$$
\operatorname{reg} I(G) \leq\left\lfloor\frac{n+1}{3}\right\rfloor+1
$$




\section{REgUlARITy AND INDUCED MATChING NUMBER OF A DUMBBELL GRAPH}

In this section we compute the induced matching number of a dumbbell graph and the regularity of its edge ideal. Recall that $C_{n} \cdot P_{l} \cdot C_{m}$ denotes the graph constructed by joining two cycles $C_{n}$ and $C_{m}$ via a path $P_{l}$. In this section, we denote the vertices of $C_{n}, C_{m}$ and $P_{l}$ by $\left\{x_{1}, \ldots, x_{n}\right\},\left\{y_{1}, \ldots, y_{m}\right\}$ and $\left\{z_{1}, \ldots, z_{l}\right\}$, respectively. We make the identifications $x_{1}=z_{1}$ and $y_{1}=z_{l}$.

Example 2.1. Two base cases when $l=2$ and $l=1$ are the following:
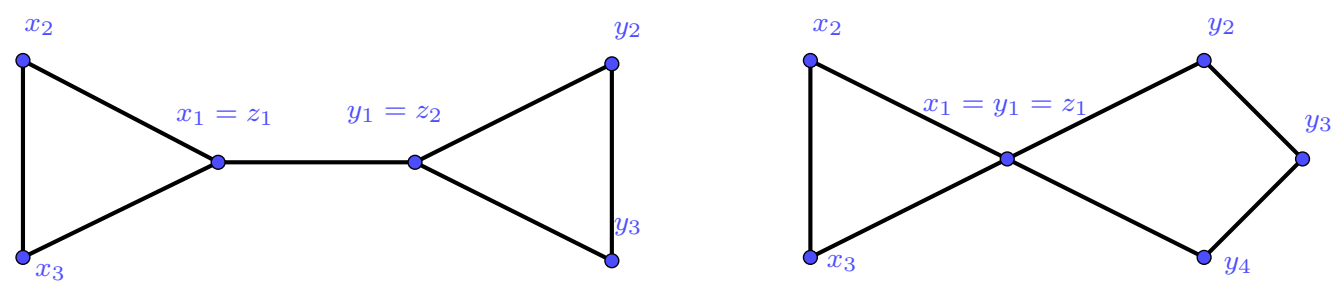

Figure 1 . The graphs $C_{3} \cdot P_{2} \cdot C_{3}$ and $C_{3} \cdot P_{1} \cdot C_{4}$.

Notation 2.2. Let $\xi_{3}$ be the function defined as below

$$
\xi_{3}(n)= \begin{cases}1 & \text { if } n \equiv 0,1(\bmod 3), \\ 0 & \text { if } n \equiv 2(\bmod 3) .\end{cases}
$$

Let $C_{n} \cdot P_{l}$ be the graph given by connecting the path $P_{l}$ to the cycle $C_{n}$. For instance, the graph $C_{3} \cdot P_{3}$ can be illustrated as the following:

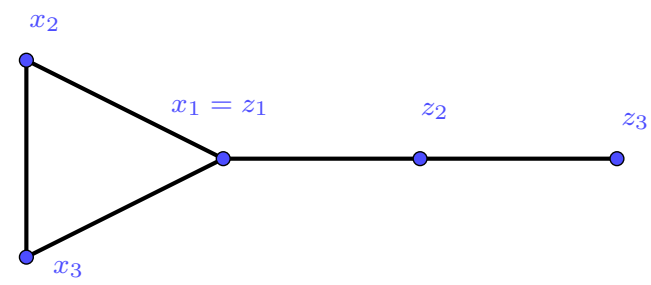

Proposition 2.3. Let $n \geq 3$ and $l \geq 1$, then

$$
\nu\left(C_{n} \cdot P_{l}\right)=\left\lfloor\frac{n}{3}\right\rfloor+\left\lfloor\frac{l-\xi_{3}(n)+1}{3}\right\rfloor .
$$

Proof. Case 1: From Remark 1.6, in the case $n \equiv 2(\bmod 3)$ we have that in clockwise and anticlockwise directions the two consecutive edges to the vertex $x_{1}$ are not chosen in a maximal induced matching of $C_{n}$. Then, we can choose the edges in $P_{l}$ without any constraint coming from the maximal induced matching chosen in $C_{n}$, and so we have $\nu\left(C_{n} \cdot P_{l}\right)=\left\lfloor\frac{n}{3}\right\rfloor+\left\lfloor\frac{l+1}{3}\right\rfloor$.

Case 2: It remains to consider the case $\xi_{3}(n)=1$, i.e., $n \equiv 0,1(\bmod 3)$. Let $\mathcal{M}$ be an induced matching of maximal size in $G$. We analyze separately the two cases of whether $z_{1} z_{2}$ (the edge adjacent to the cycle $C_{n}$ ) is in $\mathcal{M}$ or not.

Suppose $z_{1} z_{2}$ is not an edge of $\mathcal{M}$. Then $\mathcal{M}$ can be considered as the union of a maximal matching of $C_{n}$ as introduced in Remark 1.6 and a maximal matching of the path $P_{l} \backslash z_{1}$. Thus $|\mathcal{M}|=\nu\left(C_{n}\right)+\nu\left(P_{l-1}\right)=\left\lfloor\frac{n}{3}\right\rfloor+\left\lfloor\frac{(l-1)+1}{3}\right\rfloor$.

If $z_{1} z_{2} \in \mathcal{M}$, then none of the edges incident to the vertices in $N_{C_{n}}\left[x_{1}\right]=\left\{x_{1}, x_{2}, x_{n}\right\}$ are in $\left.\mathcal{M}\right|_{C_{n}}:=\left\{e \in \mathcal{M} \mid e \subset C_{n}\right\}$. Hence $|\mathcal{M}|_{C_{n}} \mid=\nu\left(P_{n-3}\right)$, and since $n \equiv 0,1(\bmod 3)$ 
then it follows $|\mathcal{M}|_{C_{n}} \mid=\left\lfloor\frac{n-2}{3}\right\rfloor=\left\lfloor\frac{n}{3}\right\rfloor-1$. From $z_{1} z_{2} \in \mathcal{M}$ we get $|\mathcal{M}|_{P_{l}} \mid=\nu\left(P_{l}\right)=\left\lfloor\frac{l+1}{3}\right\rfloor$. So, by joining both computations we get $|\mathcal{M}|=\left\lfloor\frac{n}{3}\right\rfloor-1+\left\lfloor\frac{l+1}{3}\right\rfloor=\left\lfloor\frac{n}{3}\right\rfloor+\left\lfloor\frac{l-2}{3}\right\rfloor$.

Therefore, we obtain that $\nu\left(C_{n} \cdot P_{l}\right)=\left\lfloor\frac{n}{3}\right\rfloor+\left\lfloor\frac{(l-1)+1}{3}\right\rfloor$.

Theorem 2.4. Let $n, m \geq 3$ and $l \geq 1$, then

$$
\nu\left(C_{n} \cdot P_{l} \cdot C_{m}\right)=\left\lfloor\frac{n}{3}\right\rfloor+\left\lfloor\frac{m}{3}\right\rfloor+\left\lfloor\frac{l-\xi_{3}(n)-\xi_{3}(m)+1}{3}\right\rfloor .
$$

Proof. We use the same argument as in Proposition 2.3. By Remark 1.6 we have that when either $n \equiv 2(\bmod 3)$ or $m \equiv 2(\bmod 3)$, then the maximal induced matching in $C_{n}$ or in $C_{m}$ does not affect the way we choose edges in the path $P_{l}$.

In the case $n \equiv 0,1(\bmod 3)$, we can choose a maximal induced matching that does not use the edge $z_{1} z_{2}$ by Remark 1.6, i.e., the extreme vertex $z_{1}$ on the path $P_{l}$ does not appear in the induced matching. Similarly, when $m \equiv 0,1(\bmod 3)$ we can drop the other extreme vertex.

The aim of the rest of this section is to explicitly compute the regularity of $I\left(C_{n} \cdot P_{l} \cdot C_{m}\right)$ in terms of the induced matching number. We divide it into three subsections depending on the value of $l \bmod 3$. The base of our computations is given by the following proposition.

Proposition 2.5. Let $n, m \geq 3$ and $l \geq 1$, then

$$
\operatorname{reg} I\left(C_{n} \cdot P_{l} \cdot C_{m}\right)-\nu\left(C_{n} \cdot P_{l} \cdot C_{m}\right)=\operatorname{reg} I\left(C_{n} \cdot P_{l+3} \cdot C_{m}\right)-\nu\left(C_{n} \cdot P_{l+3} \cdot C_{m}\right) .
$$

Proof. From the formula obtained in Theorem 2.4 or [25, Lemma 1], we have the equality

$$
\nu\left(C_{n} \cdot P_{l+3} \cdot C_{m}\right)=\nu\left(C_{n} \cdot P_{l} \cdot C_{m}\right)+1 .
$$

We can apply the Lozin transformation (see e.g. [25], [7]) to any of the vertices in the bridge $P_{l}$, then from [7, Theorem 1.1] we have

$$
\operatorname{reg} I\left(C_{n} \cdot P_{l+3} \cdot C_{m}\right)=\operatorname{reg} I\left(C_{n} \cdot P_{l} \cdot C_{m}\right)+1 .
$$

Thus, the statement of the proposition follows by subtracting these equalities.

From the previous proposition, it follows that we only need to consider the cases $l=1$, $l=2$ and $l=3$. We treat each case in a separate subsection.

The basic approach in the next three subsections is to obtain lower and upper bounds that coincide.

2.1. The case $l=1$. Throughout this subsection, we consider the dumbbell graph $C_{n} \cdot P_{1} \cdot C_{m}$.

Proposition 2.6. Let $n, m \geq 3$, then

$$
\operatorname{reg} I\left(C_{n} \cdot P_{1} \cdot C_{m}\right) \leq \max \left\{\left\lfloor\frac{n}{3}\right\rfloor+\left\lfloor\frac{m}{3}\right\rfloor+1,\left\lfloor\frac{n-2}{3}\right\rfloor+\left\lfloor\frac{m-2}{3}\right\rfloor+2\right\} .
$$

Moreover, $\operatorname{reg} I\left(C_{n} \cdot P_{1} \cdot C_{m}\right)$ is equal to one of these terms.

Proof. We use [11, Lemma 3.2] that gives an improved version of the exact sequence that comes from deleting the vertex $z_{1}$ and its neighbors. We have

$$
\operatorname{reg} I\left(C_{n} \cdot P_{1} \cdot C_{m}\right) \in\left\{\operatorname{reg} I\left(\left(C_{n} \cdot P_{1} \cdot C_{m}\right) \backslash z_{1}\right), \operatorname{reg} I\left(\left(C_{n} \cdot P_{1} \cdot C_{m}\right) \backslash N\left[z_{1}\right]\right)+1\right\} .
$$

Since $\left(C_{n} \cdot P_{1} \cdot C_{m}\right) \backslash z_{1}=P_{n-1} \cup P_{m-1}$ and $\left(C_{n} \cdot P_{1} \cdot C_{m}\right) \backslash N\left[z_{1}\right]=P_{n-3} \cup P_{m-3}$, we get the result by applying Theorem 1.15 . 
Theorem 2.7. Let $n, m \geq 3$, then

$$
\operatorname{reg} I\left(C_{n} \cdot P_{1} \cdot C_{m}\right)= \begin{cases}\nu\left(C_{n} \cdot P_{1} \cdot C_{m}\right)+2 & \text { if } n \equiv 2(\bmod 3), m \equiv 2(\bmod 3) ; \\ \nu\left(C_{n} \cdot P_{1} \cdot C_{m}\right)+1 & \text { otherwise }\end{cases}
$$

Proof. Suppose $n \equiv 2(\bmod 3)$ and $m \equiv 2(\bmod 3)$. Since $\left\lfloor\frac{k-2}{3}\right\rfloor=\left\lfloor\frac{k}{3}\right\rfloor$ when $k \equiv$ $2(\bmod 3)$, we have

$$
\max \left\{\left\lfloor\frac{n}{3}\right\rfloor+\left\lfloor\frac{m}{3}\right\rfloor+1,\left\lfloor\frac{n-2}{3}\right\rfloor+\left\lfloor\frac{m-2}{3}\right\rfloor+2\right\}=\left\lfloor\frac{n}{3}\right\rfloor+\left\lfloor\frac{m}{3}\right\rfloor+2 .
$$

Thus Proposition 2.6 yields

$$
\operatorname{reg} I\left(C_{n} \cdot P_{1} \cdot C_{m}\right) \leq\left\lfloor\frac{n}{3}\right\rfloor+\left\lfloor\frac{m}{3}\right\rfloor+2 .
$$

Consider the induced subgraph $H=\left(C_{n} \cdot P_{1} \cdot C_{m}\right) \backslash\left\{x_{n}\right\}$ where $x_{n}$ is in $C_{n}$ and it is incident to $x_{1}$ (e.g. see $x_{3}$ in Example 2.1). In fact, $H$ is the graph given by joining $C_{m}$ and a path $P_{n-1}$, that is, $H=C_{m} \cdot P_{n-1}$. Now from Proposition 2.3, we have that $\nu(H)=\left\lfloor\frac{n}{3}\right\rfloor+\left\lfloor\frac{m}{3}\right\rfloor$. By Theorem 1.7 $(i)$, we get $\operatorname{reg} I\left(C_{n} \cdot P_{1} \cdot C_{m}\right) \geq \operatorname{reg} I(H)$. From [2, Theorem 1.2], we have $\operatorname{reg} I(H)=\nu(H)+2$. Therefore, the equality holds in (1). The proof of this part is complete since Theorem 2.4 yields $\nu\left(C_{n} \cdot P_{1} \cdot C_{m}\right)=\left\lfloor\frac{n}{3}\right\rfloor+\left\lfloor\frac{m}{3}\right\rfloor$.

For any case distinct to $n \equiv 2(\bmod 3)$ and $m \equiv 2(\bmod 3)$, we have

$$
\max \left\{\left\lfloor\frac{n}{3}\right\rfloor+\left\lfloor\frac{m}{3}\right\rfloor+1,\left\lfloor\frac{n-2}{3}\right\rfloor+\left\lfloor\frac{m-2}{3}\right\rfloor+2\right\}=\left\lfloor\frac{n}{3}\right\rfloor+\left\lfloor\frac{m}{3}\right\rfloor+1 .
$$

Therefore, from Proposition 2.6, we have

$$
\operatorname{reg} I\left(C_{n} \cdot P_{1} \cdot C_{m}\right) \leq\left\lfloor\frac{n}{3}\right\rfloor+\left\lfloor\frac{m}{3}\right\rfloor+1 .
$$

From Theorem 2.4, we have $\nu\left(C_{n} \cdot P_{1} \cdot C_{m}\right)=\left\lfloor\frac{n}{3}\right\rfloor+\left\lfloor\frac{m}{3}\right\rfloor$. Moreover, Theorem 1.16 gives $\operatorname{reg} I\left(C_{n} \cdot P_{1} \cdot C_{m}\right) \geq \nu\left(C_{n} \cdot P_{1} \cdot C_{m}\right)+1$. Thus, the equality in (2) holds. Therefore the proof is complete.

2.2. The case $l=2$. Throughout this subsection, we consider the dumbbell graph $C_{n} \cdot P_{2} \cdot C_{m}$.

Remark 2.8. The regularity of $I\left(C_{n}\right)$ is given in Theorem 1.20. For simplicity of notation, we use the equivalent formula reg $I\left(C_{n}\right)=\left\lfloor\frac{n-2}{3}\right\rfloor+2$. Similarly, we have $\operatorname{reg}\left(R / I\left(C_{n}\right)\right)=$ $\left\lfloor\frac{n-2}{3}\right\rfloor+1$.

Proposition 2.9. Let $n, m \geq 3$, then

$$
\nu\left(C_{n} \cdot P_{2} \cdot C_{m}\right) \leq \operatorname{reg}\left(\frac{R}{I\left(C_{n} \cdot P_{2} \cdot C_{m}\right)}\right) \leq\left\lfloor\frac{n-2}{3}\right\rfloor+\left\lfloor\frac{m-2}{3}\right\rfloor+2 .
$$

Proof. We only need to prove the inequality on the right since the lower bound is given due to Theorem 1.16. In the original graph $C_{n} \cdot P_{2} \cdot C_{m}$ we remove the edge that connects the two cycles $C_{n}$ and $C_{m}$. The set of vertices of $C_{n}$ and $C_{m}$ are given respectively by $\left\{x_{1}, \ldots, x_{n}\right\}$ and $\left\{y_{1}, \ldots, y_{m}\right\}$, and we assume that the edge $e=x_{1} y_{1}$ is the bridge between the two cycles. We denote by $C_{n} \cup C_{m}$ the resulting graph given as the disjoint union of the two cycles $C_{n}$ and $C_{m}$.

Note that $\left(C_{n} \cdot P_{2} \cdot C_{m}\right) \backslash e=C_{n} \cup C_{m}$ and that $I\left(\left(C_{n} \cdot P_{2} \cdot C_{m}\right)_{e}\right)=I\left(P_{n-3} \cup P_{m-3}\right)$ because $N_{G}\left[x_{1}\right] \cup N_{G}\left[y_{1}\right]=\left\{x_{1}, x_{2}, x_{n}, y_{1}, y_{2}, y_{m}\right\}$, where $P_{n-3}$ is the path on the vertices 
$\left\{x_{3}, \ldots, x_{n-1}\right\}$ and $P_{m-3}$ is the path on the vertices $\left\{y_{3}, \ldots, y_{m-1}\right\}$. Thus, Theorem $1.7($ iii $)$ gives the inequality

$$
\operatorname{reg}\left(\frac{R}{I\left(C_{n} \cdot P_{2} \cdot C_{m}\right)}\right) \leq \max \left\{\operatorname{reg}\left(\frac{R}{I\left(C_{n} \cup C_{m}\right)}\right), \operatorname{reg}\left(\frac{R}{I\left(P_{n-3} \cup P_{m-3}\right)}\right)+1\right\} .
$$

From Corollary 1.14 and Remark 2.8, it follows that

$$
\operatorname{reg}\left(\frac{R}{I\left(C_{n} \cup C_{m}\right)}\right)=\left\lfloor\frac{n-2}{3}\right\rfloor+\left\lfloor\frac{m-2}{3}\right\rfloor+2 .
$$

Similarly, Theorem 1.15 and Remark 1.5 give that

$$
\operatorname{reg}\left(\frac{R}{I\left(P_{n-3} \cup P_{m-3}\right)}\right)+1=\left\lfloor\frac{n-2}{3}\right\rfloor+\left\lfloor\frac{m-2}{3}\right\rfloor+1 .
$$

This proves the proposition.

As a result of the previous proposition, we can prove the following corollary.

Corollary 2.10. If $n \equiv 0,1(\bmod 3)$ and $m \equiv 0,1(\bmod 3)$, then

$$
\operatorname{reg}\left(\frac{R}{I\left(C_{n} \cdot P_{2} \cdot C_{m}\right)}\right)=\nu\left(C_{n} \cdot P_{2} \cdot C_{m}\right)=\left\lfloor\frac{n}{3}\right\rfloor+\left\lfloor\frac{m}{3}\right\rfloor
$$

Proof. Note that $\left\lfloor\frac{k}{3}\right\rfloor=\left\lfloor\frac{k-2}{3}\right\rfloor+1$ when $k \equiv 0,1(\bmod 3)$. From Theorem 2.4, in $(3)$ the lower and upper bound coincide for these cases. So the equality is established.

Now we have only three more cases left to deal with, i.e., the case $n \equiv 0(\bmod 3), m \equiv$ $2(\bmod 3)$, the case $n \equiv 1(\bmod 3), m \equiv 2(\bmod 3)$, and the case $n \equiv 2(\bmod 3), m \equiv$ $2(\bmod 3)$.

Lemma 2.11. If $n \equiv 2(\bmod 3)$ and $m \equiv 2(\bmod 3)$, then

$$
\operatorname{reg}\left(\frac{R}{I\left(C_{n} \cdot P_{2} \cdot C_{m}\right)}\right)=\nu\left(C_{n} \cdot P_{2} \cdot C_{m}\right)=\left\lfloor\frac{n}{3}\right\rfloor+\left\lfloor\frac{m}{3}\right\rfloor+1 .
$$

Proof. We shall divide the graph into three subgraphs $H_{1}, H_{2}$ and $H_{3}$. We make $H_{1}=$ $C_{n} \backslash\left\{x_{1}\right\}$ and $H_{2}=C_{m} \backslash\left\{y_{1}\right\}$. The subgraph $H_{3}$ is defined by taking the bridge $e=x_{1} y_{1}$ and the neighboring vertices $\left\{x_{2}, x_{n}, y_{2}, y_{m}\right\}$, i.e. the graph below.

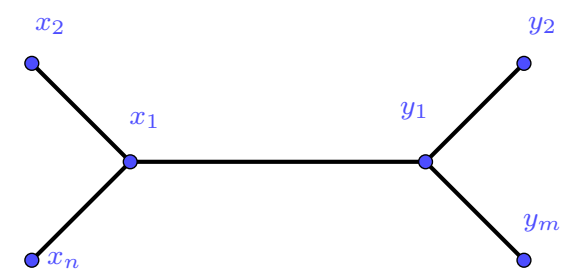

Using this decomposition and Theorem 1.12 we get the inequality

$$
\operatorname{reg} R / I\left(C_{n} \cdot P_{2} \cdot C_{m}\right) \leq \operatorname{reg}\left(R / I\left(H_{1}\right)\right)+\operatorname{reg}\left(R / I\left(H_{2}\right)\right)+\operatorname{reg}\left(R / I\left(H_{3}\right)\right),
$$

where $H_{1}$ and $H_{2}$ are paths of length $n-1$ and $m-1$, respectively, and using Theorem 1.15 we get

$$
\operatorname{reg} R / I\left(C_{n} \cdot P_{2} \cdot C_{m}\right) \leq\left\lfloor\frac{n}{3}\right\rfloor+\left\lfloor\frac{m}{3}\right\rfloor+1 .
$$

Finally, in the present case $n \equiv 2(\bmod 3)$ and $m \equiv 2(\bmod 3)$ we have the equality $\nu\left(C_{n} \cdot P_{2} \cdot C_{m}\right)=\left\lfloor\frac{n}{3}\right\rfloor+\left\lfloor\frac{m}{3}\right\rfloor+1$, and so the proof follows from Theorem 1.16. 
Lemma 2.12. If $n \equiv 0,1(\bmod 3)$ and $m \equiv 2(\bmod 3)$, then

$$
\operatorname{reg}\left(\frac{R}{I\left(C_{n} \cdot P_{2} \cdot C_{m}\right)}\right)=\nu\left(C_{n} \cdot P_{2} \cdot C_{m}\right)+1=\left\lfloor\frac{n}{3}\right\rfloor+\left\lfloor\frac{m}{3}\right\rfloor+1 .
$$

Proof. In this case we delete the vertex $x_{1}$ from the cycle $C_{n}$. We have that $H=$ $\left(C_{n} \cdot P_{2} \cdot C_{m}\right) \backslash\left\{x_{1}\right\}$ is an induced subgraph of $C_{n} \cdot P_{2} \cdot C_{m}$ which is given as the disjoint union of a path of length $n-1$ and $C_{m}$, i.e. $H=P_{n-1} \cup C_{m}$. From Theorem 1.7 $(i)$, Corollary 1.14, Theorem 1.15 and Theorem 1.20 we get that

$$
\operatorname{reg}\left(R / I\left(C_{n} \cdot P_{2} \cdot C_{m}\right)\right) \geq \operatorname{reg}(R / I(H))=\left\lfloor\frac{n}{3}\right\rfloor+\left\lfloor\frac{m}{3}\right\rfloor+1 .
$$

It follows from Proposition 2.9 and the fact that $\lfloor k / 3\rfloor=\lfloor(k-2) / 3\rfloor+1$ when $k \equiv 0,1(\bmod 3)$ that

$$
\operatorname{reg} R / I\left(C_{n} \cdot P_{2} \cdot C_{m}\right)=\left\lfloor\frac{n}{3}\right\rfloor+\left\lfloor\frac{m}{3}\right\rfloor+1 .
$$

So we are through.

Theorem 2.13. Let $n, m \geq 3$, then

$$
\operatorname{reg} I\left(C_{n} \cdot P_{2} \cdot C_{m}\right)= \begin{cases}\nu\left(C_{n} \cdot P_{2} \cdot C_{m}\right)+2 & \text { if } n \equiv 0,1(\bmod 3), m \equiv 2(\bmod 3) ; \\ \nu\left(C_{n} \cdot P_{2} \cdot C_{m}\right)+1 & \text { otherwise. }\end{cases}
$$

Proof. It follows from Corollary 2.10, Lemma 2.11 and Lemma 2.12.

2.3. The case $l=3$. Throughout this subsection, we consider the dumbbell graph $C_{n} \cdot P_{3} \cdot C_{m}$.

Proposition 2.14. Let $n, m \geq 3$, then

(i) $\operatorname{reg} I\left(C_{n} \cdot P_{3} \cdot C_{m}\right) \leq \nu\left(C_{n} \cdot P_{3} \cdot C_{m}\right)+2$, if $n, m \equiv 2(\bmod 3)$;

(ii) $\operatorname{reg} I\left(C_{n} \cdot P_{3} \cdot C_{m}\right)=\nu\left(C_{n} \cdot P_{3} \cdot C_{m}\right)+1$, otherwise.

Proof. Let $E\left(P_{3}\right)=\left\{e, e^{\prime}\right\}$ be the set of edges of $P_{3}$, where $e=z_{1} z_{2}$ and $e^{\prime}=z_{2} z_{3}$ are connected to $C_{n}$ and $C_{m}$, respectively. Note that $I\left(\left(C_{n} \cdot P_{3} \cdot C_{m}\right) \backslash e\right)=I\left(C_{n} \cup\left(e^{\prime} \cdot C_{m}\right)\right)$ and that $I\left(\left(C_{n} \cdot P_{3} \cdot C_{m}\right)_{e}\right)=I\left(P_{n-3} \cup P_{m-1}\right)$ because

$$
N_{G}[e]=\left\{x_{1}=z_{1}, x_{2}, x_{n}, z_{2}, y_{1}=z_{3}\right\},
$$

where $e^{\prime} \cdot C_{m}$ is the unicyclic graph with $C_{m}$ and a whisker $e^{\prime}$ attached to $C_{m}, P_{n-3}$ is the path on the vertices $\left\{x_{3}, \ldots, x_{n-1}\right\}$ and $P_{m-1}$ is the path on the vertices $\left\{y_{2}, \ldots, y_{m}\right\}$. Thus, Theorem 1.7(iii) gives the inequality

$$
\operatorname{reg}\left(\frac{R}{I\left(C_{n} \cdot P_{3} \cdot C_{m}\right)}\right) \leq \max \left\{\operatorname{reg}\left(\frac{R}{I\left(C_{n} \cup\left(e^{\prime} \cdot C_{m}\right)\right.}\right), \operatorname{reg}\left(\frac{R}{I\left(P_{n-3} \cup P_{m-1}\right)}\right)+1\right\} .
$$

From Proposition 2.3 and [2, Lemma 3.2] follows that reg $\left(I\left(e^{\prime} \cdot C_{m}\right)\right)=\left\lfloor\frac{m}{3}\right\rfloor+\left\lfloor\frac{3-\xi_{3}(m)}{3}\right\rfloor+$ 1. Thus, using Remark 2.8, Corollary 1.14 and Theorem 1.15, we get reg $\left(\frac{R}{I\left(C_{n} \cdot P_{3} \cdot C_{m}\right)}\right) \leq$ $\max \left\{\left\lfloor\frac{n-2}{3}\right\rfloor+1+\left\lfloor\frac{m}{3}\right\rfloor+\left\lfloor\frac{3-\xi_{3}(m)}{3}\right\rfloor,\left\lfloor\frac{n-2}{3}\right\rfloor+\left\lfloor\frac{m}{3}\right\rfloor+1\right\}$.

On the other hand, from Theorem 2.4 we have that $\nu\left(C_{n} \cdot P_{3} \cdot C_{m}\right)=\left\lfloor\frac{n}{3}\right\rfloor+\left\lfloor\frac{m}{3}\right\rfloor+$ $\left\lfloor\frac{4-\xi_{3}(n)-\xi_{3}(m)}{3}\right\rfloor$. Therefore, we see that $\operatorname{reg}\left(\frac{R}{I\left(C_{n} \cdot P_{3} \cdot C_{m}\right)}\right) \leq \nu\left(C_{n} \cdot P_{3} \cdot C_{m}\right)+1$ when $n, m \equiv 2(\bmod 3)$, and that $\operatorname{reg}\left(\frac{R}{I\left(C_{n} \cdot P_{3} \cdot C_{m}\right)}\right)=\nu\left(C_{n} \cdot P_{3} \cdot C_{m}\right)$ in all the remaining cases. 
Theorem 2.15. Let $n, m \geq 3$, then

$$
\operatorname{reg} I\left(C_{n} \cdot P_{3} \cdot C_{m}\right)= \begin{cases}\nu\left(C_{n} \cdot P_{3} \cdot C_{m}\right)+2 & \text { if } n, m \equiv 2(\bmod 3), \\ \nu\left(C_{n} \cdot P_{3} \cdot C_{m}\right)+1 & \text { otherwise. }\end{cases}
$$

Proof. From Proposition 2.14, it suffices to show that reg $I\left(C_{n} \cdot P_{3} \cdot C_{m}\right) \geq \nu\left(C_{n} \cdot P_{3} \cdot C_{m}\right)+2$ when $n, m \equiv 2(\bmod 3)$. Hence, we assume $n, m \equiv 2(\bmod 3)$. Let $z_{2}$ be the middle vertex of $C_{n} \cdot P_{3} \cdot C_{m}$. By deleting $z_{2}$ we see that $H=\left(C_{n} \cdot P_{3} \cdot C_{m}\right) \backslash z_{2}=C_{n} \cup C_{m}$ is an induced subgraph of $C_{n} \cdot P_{3} \cdot C_{m}$. From Theorem 1.20 and Corollary 1.14, we have that

$$
\operatorname{reg} I(H)=\operatorname{reg} I\left(C_{n}\right)+\operatorname{reg} I\left(C_{m}\right)-1=\nu\left(C_{n}\right)+\nu\left(C_{m}\right)+3 .
$$

Since $\nu\left(C_{n} \cdot P_{3} \cdot C_{m}\right)=\nu\left(C_{n}\right)+\nu\left(C_{m}\right)+1$, then using Theorem 1.7(i) we get

$$
\operatorname{reg} I\left(C_{n} \cdot P_{3} \cdot C_{m}\right) \geq \operatorname{reg} I(H)=\nu\left(C_{n} \cdot P_{3} \cdot C_{m}\right)+2 .
$$

2.4. Regularity of a dumbbell graph. Now we are ready for the main result of this section. In the following theorem we compute the regularity of the edge ideal of the dumbbell $C_{n} \cdot P_{l} \cdot C_{m}$.

Theorem 2.16. Let $m, n \geq 3$ and $l \geq 1$, then

(i) if $l \equiv 0,1(\bmod 3)$, then

$$
\operatorname{reg} I\left(C_{n} \cdot P_{l} \cdot C_{m}\right)= \begin{cases}\nu\left(C_{n} \cdot P_{l} \cdot C_{m}\right)+2 & \text { if } n, m \equiv 2(\bmod 3), \\ \nu\left(C_{n} \cdot P_{l} \cdot C_{m}\right)+1 & \text { otherwise; }\end{cases}
$$

(ii) if $l \equiv 2(\bmod 3)$, then

$$
\operatorname{reg} I\left(C_{n} \cdot P_{l} \cdot C_{m}\right)= \begin{cases}\nu\left(C_{n} \cdot P_{l} \cdot C_{m}\right)+2 & n \equiv 0,1(\bmod 3), m \equiv 2(\bmod 3) ; \\ \nu\left(C_{n} \cdot P_{l} \cdot C_{m}\right)+1 & \text { otherwise. }\end{cases}
$$

Proof. Follows from Proposition 2.5, and Theorem 2.7, Theorem 2.13, and Theorem 2.15.

\section{Combinatorial Characterization of reg $(I(G))$ in terms of $\nu(G)$}

In this section, we focus on any bicyclic graph which admits the dumbbell graph $C_{n} \cdot P_{l} \cdot C_{m}$ as its base bicycle and study the regularity of the edge ideals of these graphs.

Let $G$ be any bicyclic graph with dumbbell $C_{n} \cdot P_{l} \cdot C_{m}$. Then its decycling number is smaller or equal than 2. Thus by Theorem 1.16 and Theorem 1.18, we get

$$
\nu(G)+1 \leq \operatorname{reg} I(G) \leq \nu(G)+3 .
$$

There are examples of bicyclic graphs with a dumbbell where the regularity of its edge ideal is equal to $\nu(G)+1, \nu(G)+2$ and $\nu(G)+3$.

Example 3.1. The following graph $G$

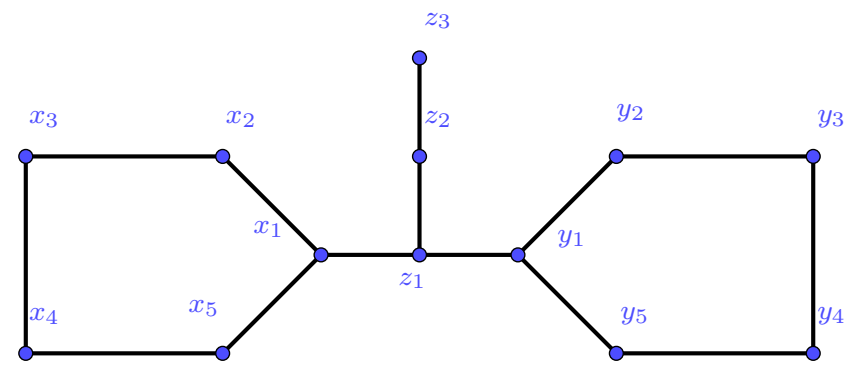

has $\operatorname{reg} I(G)=6$ and $\nu(G)=3$. 
In this section, we give a combinatorial characterization of the bicyclic graphs with regularity $\nu(G)+1, \nu(G)+2$ and $\nu(G)+3$.

For the rest of the paper, we shall use the term "dumbbell" of the bicyclic graph $G$, and it denotes the unique subgraph of $G$ of the form $C_{n} \cdot P_{l} \cdot C_{m}$.

The following simple remark will be crucial in our treatment.

Remark 3.2. [2, Observation 2.1] Let $G$ be a graph with a leaf $y$ and its unique neighbor $x$, say $e=\{x, y\}$. If $\left\{e_{1}, \ldots, e_{s}\right\}$ is an induced matching in $G \backslash N[x]$, then $\left\{e_{1}, \ldots, e_{s}, e\right\}$ is an induced matching in $G$. So we have $\nu(G \backslash N[x])+1 \leq \nu(G)$.

Proposition 3.3. Let $G$ be a bicyclic graph with dumbbell $C_{n} \cdot P_{l} \cdot C_{m}$. The following statements hold.

(i) When $n, m \equiv 0,1(\bmod 3)$, we have $\operatorname{reg} I(G)=\nu(G)+1$.

(ii) When $n \equiv 0,1(\bmod 3)$ and $m \equiv 2(\bmod 3)$, we have $\operatorname{reg} I(G) \leq \nu(G)+2$.

(iii) When $l \leq 2$, we have $\operatorname{reg} I(G) \leq \nu(G)+2$.

Proof. (i) Again, it is enough to prove the upper bound $\operatorname{reg} I(G) \leq \nu(G)+1$. Let $E^{\prime}$ be the set of edges $E^{\prime}=E(G) \backslash E\left(C_{n} \cdot P_{l} \cdot C_{m}\right)$. We proceed by induction on the cardinality of $E^{\prime}$. If $\left|E^{\prime}\right|=0$ then the statement follows from Theorem 2.16, so we assume $\left|E^{\prime}\right|>0$. There exists a leaf $y$ in $G$ such that $N[y]=\{x\}$. Let $G^{\prime}=G \backslash x$ and $G^{\prime \prime}=G \backslash N[x]$, then by Theorem 1.7 we have

$$
\operatorname{reg} I(G) \leq \max \left\{\operatorname{reg} I\left(G^{\prime}\right), \operatorname{reg} I\left(G^{\prime \prime}\right)+1\right\} .
$$

The graphs $G^{\prime}$ and $G^{\prime \prime}$ can be either bicyclic graphs with the same dumbbell $C_{n} \cdot P_{l} \cdot C_{m}$, or the disjoint union of two unicyclic graphs with cycles $C_{n}$ and $C_{m}$, or unicyclic graphs with a cycle $C_{r}(r=n$ or $r=m)$ of the type $r \equiv 0,1(\bmod 3)$, or forests. Using either the induction hypothesis, or [2, Theorem 1.2] and Corollary 1.14, or [2, Theorem 1.2], or Theorem 1.15, then we get $\operatorname{reg} I\left(G^{\prime}\right)=\nu\left(G^{\prime}\right)+1$ and $\operatorname{reg} I\left(G^{\prime \prime}\right)=\nu\left(G^{\prime \prime}\right)+1$. Since we have $\nu\left(G^{\prime}\right) \leq \nu(G)$ and $\nu\left(G^{\prime \prime}\right)+1 \leq \nu(G)$ (by Remark 3.2), then we obtain the required inequality.

(ii) and (iii) follow by the same inductive argument, only changing the fact that $G^{\prime}$ and $G^{\prime \prime}$ could be unicyclic graphs with cycle $C_{r}$ of the type $r \equiv 2(\bmod 3)$.

Remark 3.4. The inductive process of the previous proposition cannot conclude $\operatorname{reg} I(G) \leq$ $\nu(G)+2$ in the case $l \geq 3$. Here we may encounter two disjoint induced subgraphs $G_{1}$ and $G_{2}$ with $\operatorname{reg} I\left(G_{i}\right)=\nu\left(G_{i}\right)+2$, which implies $\operatorname{reg} I\left(G_{1} \cup G_{2}\right)=\nu\left(G_{1} \cup G_{2}\right)+3$. This is exactly the case of Example 3.1.

An alternative proof of the inequality $\operatorname{reg} I(G) \leq \nu(G)+3$ for $l \geq 3$ can be given by using the same inductive technique of Proposition 3.3.

For the rest of the paper we use the following notation.

Notation 3.5. Let $G$ be a graph, $H \subset G$ be a subgraph, and $v$ and $w$ be vertices of $G$. Then, we assume the following:

(i) $d(v, w)$ denotes the length (i.e., the number of edges) of a minimal path between $v$ and $w$. In particular, $d(v, v)=0$.

(ii) $d(v, H)$ denotes the minimal distance from the vertex $v$ to the subgraph $H$, that is

$$
d(v, H)=\min \{d(v, w) \mid w \in H\} .
$$

In particular, $d(v, H)=0$ if and only if $v \in H$.

(iii) Let $H^{\prime} \subset G$ be a subgraph, then the distance between $H$ and $H^{\prime}$ is given by

$$
d\left(H, H^{\prime}\right)=\min \left\{d\left(v, H^{\prime}\right) \mid v \in H\right\} .
$$

In particular, $d\left(H, H^{\prime}\right)=0$ if and only if $H \cap H^{\prime} \neq \emptyset$. 
(iv) $\Gamma_{G}(H)$ denotes the subset of vertices

$$
\Gamma_{G}(H)=\{v \in G \mid d(v, H)=1\} .
$$

(v) In the case $k>0, S_{G, k}(H)$ denotes the induced subgraph given by restricting to the vertex set

$$
V\left(S_{G, k}(H)\right)=\{v \in G \mid d(v, H) \geq k\} .
$$

(vi) $S_{G, 0}(H)$ denotes the subgraph given by the vertex set

$$
V\left(S_{G, 0}(H)\right)=\{v \in G \mid d(v, H)>0 \text { or } \operatorname{deg}(v) \geq 3\} .
$$

and the edge set

$$
\begin{aligned}
E\left(S_{G, 0}(H)\right)=\{(v, w) \in E(G) & \left.\mid v, w \in V\left(S_{G, 0}(H)\right)\right\} \\
& \backslash\{(v, w) \in E(G) \mid v, w \in H\} .
\end{aligned}
$$

We clarify the previous notation in the following example.

Example 3.6. (i) Let $G$ be the graph of Example 3.1 and $H=C_{5} \cup C_{5}$ be the subgraph given by the two cycles of length 5 . Then, we have that $\Gamma_{G}(H)$ is the set containing the vertex in the middle of the bridge joining the two cycles, that $S_{G, 0}(H)$ is a graph of the form

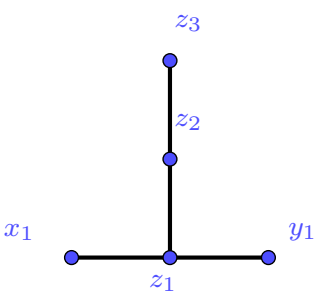

and that the graph

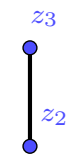

represents $S_{G, 2}(H)$.

(ii) Let $G$ be the graph given by

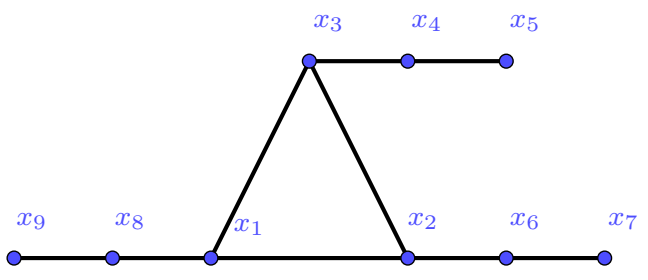

and $H$ be the triangle induced by the vertices $\left\{x_{1}, x_{2}, x_{3}\right\}$. Then, we have that $\Gamma_{G}(H)=\left\{x_{4}, x_{6}, x_{8}\right\}$, that $S_{G, 0}(H)$ is a graph of the form
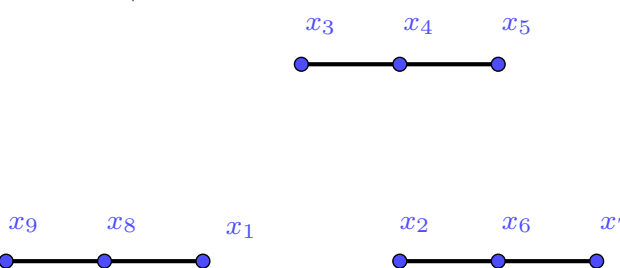

and that the graph 


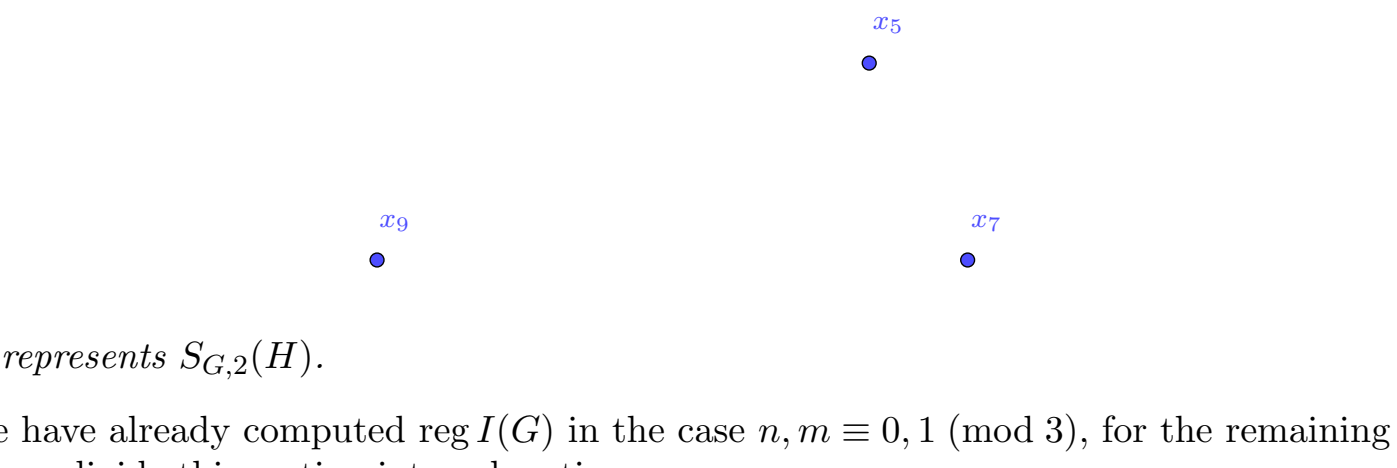

We have already computed $\operatorname{reg} I(G)$ in the case $n, m \equiv 0,1(\bmod 3)$, for the remaining cases we divide this section into subsections.

3.1. Case I. In this subsection we focus on the case $n \equiv 0,1(\bmod 3)$ and $m \equiv 2(\bmod 3)$. This case turns out to be almost identical to a unicyclic graph, and our treatment is influenced by [2, Section 3].

Notation 3.7. Let $G$ be a bicyclic graph with dumbbell $C_{n} \cdot P_{l} \cdot C_{m}$ such that $n \equiv 0,1(\bmod 3)$ and $m \equiv 2(\bmod 3)$. We denote by $F_{1}, \ldots, F_{c}$ the connected components of $S_{G, 0}\left(C_{m}\right)$, and in this case each $F_{i}$ is either a tree or a unicyclic graph with cycle $C_{n}($ and $n \equiv 0,1(\bmod 3))$. Then, the graph $S_{G, 2}\left(C_{m}\right)$ can be given as the union of the components $H_{1}, \ldots, H_{c}$, where each one is defined as

$$
H_{i}=F_{i} \backslash\left\{v \in G \mid d\left(v, C_{m}\right) \leq 1\right\} .
$$

Note that each $H_{i}$ can be a disconnected graph or even the empty graph.

Remark 3.8. The following statements hold.

(i) The graph $G \backslash \Gamma_{G}\left(C_{m}\right)$ has a decomposition of the form

$$
G \backslash \Gamma_{G}\left(C_{m}\right)=C_{m} \bigcup\left(\bigcup_{i=1}^{c} H_{i}\right),
$$

and in particular

$$
\nu\left(G \backslash \Gamma_{G}\left(C_{m}\right)\right)=\nu\left(C_{m}\right)+\sum_{i=1}^{c} \nu\left(H_{i}\right)
$$

because $d\left(C_{m}, H_{i}\right) \geq 2$ for all $1 \leq i \leq c$ and $d\left(H_{i}, H_{j}\right) \geq 2$ for all $1 \leq i<j \leq c$.

(ii) For each $i=1, \ldots, c$, we have that $\left|F_{i} \cap C_{m}\right|=1$.

Example 3.9. Let $G$ be the graph

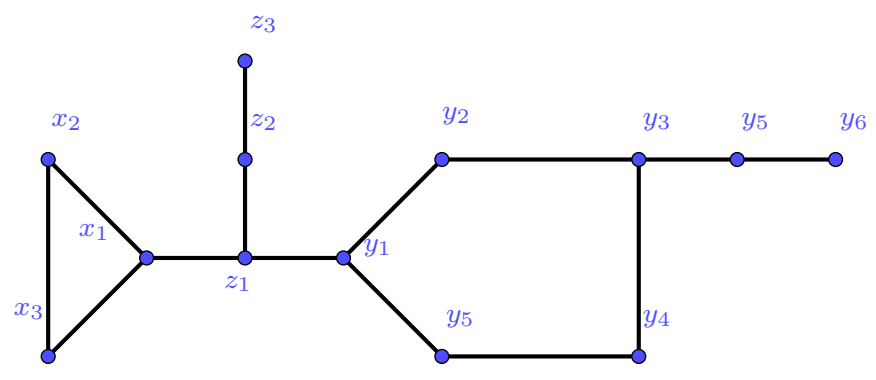

and $C_{5}$ be the cycle given by $\left\{y_{1}, y_{2}, y_{3}, y_{4}, y_{5}\right\}$. We have that $\Gamma_{G}\left(C_{5}\right)=\left\{z_{1}, y_{5}\right\}$. The graph $S_{G, 0}\left(C_{5}\right)$ is given by 

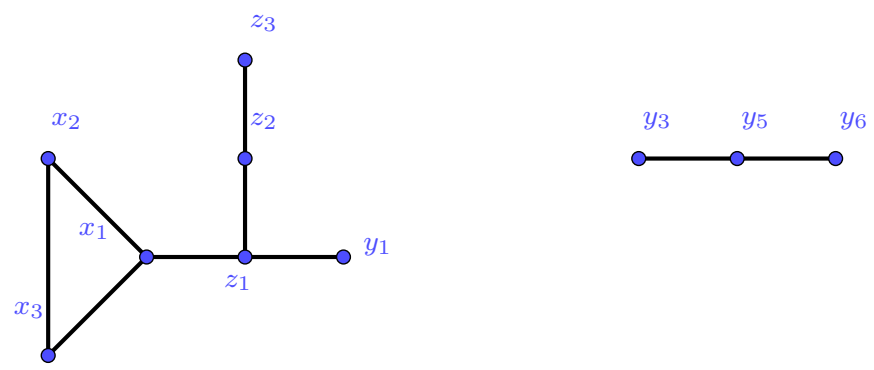

with connected components $F_{1}$ (graph on the left) and $F_{2}$ (graph on the right). The graph $S_{G, 2}\left(C_{5}\right)$ is given by

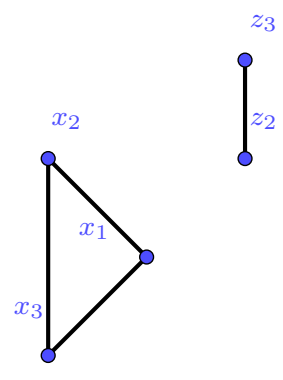

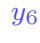

with connected components $H_{1}$ (graph on the left) and $H_{2}$ (graph on the right).

Lemma 3.10. Adopt Notation 3.7. If $\nu\left(H_{i}\right)=\nu\left(F_{i}\right)$ for all $1 \leq i \leq c$, then $\nu(G \backslash$ $\left.\Gamma_{G}\left(C_{m}\right)\right)=\nu(G)$.

Proof. Follows identically to [2, Lemma 3.5].

Proposition 3.11. Adopt Notation 3.7. If $\nu\left(G \backslash \Gamma_{G}\left(C_{m}\right)\right)<\nu(G)$ then $\operatorname{reg} I(G)=\nu(G)+1$.

Proof. Once more, we shall only prove that $\operatorname{reg} I(G) \leq \nu(G)+1$. Assume that $\nu(G \backslash$ $\left.\Gamma_{G}\left(C_{m}\right)\right)<\nu(G)$, then the contrapositive of Lemma 3.10 implies that there exists some $i$ with $\nu\left(H_{i}\right)<\nu\left(F_{i}\right)$.

Fix $i$ such that $\nu\left(H_{i}\right)<\nu\left(F_{i}\right)$. From Remark 3.8(ii), let $x$ be the vertex in $F_{i} \cap C_{m}$. Let us use the notations $G^{\prime}=G \backslash x$ and $G^{\prime \prime}=G \backslash N[x]$. Again, we have the inequality

$$
\operatorname{reg} I(G) \leq \max \left\{\operatorname{reg} I\left(G^{\prime}\right), \operatorname{reg} I\left(G^{\prime \prime}\right)+1\right\} .
$$

Note that both $G^{\prime}$ and $G^{\prime \prime}$ can be either unicyclic graphs with cycle $C_{n}$ (and $n \equiv$ $0,1(\bmod 3))$, or forests. Hence, from [2, Theorem 1.2] and Theorem 1.15 we get that $\operatorname{reg} I\left(G^{\prime}\right)=\nu\left(G^{\prime}\right)+1$ and $\operatorname{reg} I\left(G^{\prime \prime}\right)=\nu\left(G^{\prime \prime}\right)+1$.

In the case of $G^{\prime}$, we have that $\operatorname{reg} I\left(G^{\prime}\right)=\nu\left(G^{\prime}\right)+1 \leq \nu(G)+1$. Let $H$ be the induced subgraph of $G$ obtained by deleting the vertices of $F_{i} \cup N_{G}[x]$. Then we have $G^{\prime \prime}=H \cup H_{i}$. Let $\mathcal{M}_{1}$ and $\mathcal{M}_{2}$ be maximal induced matchings in $H$ and $H_{i}$, respectively, then $\nu\left(G^{\prime \prime}\right)=\left|\mathcal{M}_{1}\right|+\left|\mathcal{M}_{2}\right|$ because $d\left(H, H_{i}\right) \geq 2$. By the condition $\nu\left(F_{i}\right)>\nu\left(H_{i}\right)$ then there exists a maximal induced matching $\mathcal{M}_{3}$ in $F_{i}$, such that $\left|\mathcal{M}_{3}\right|>\left|\mathcal{M}_{2}\right|$. From the fact that $H \cup F_{i}$ is an induced subgraph in $G$ and $d\left(H, F_{i}\right) \geq 2$, then we get

$$
\nu(G) \geq \nu\left(H \cup F_{i}\right)=\left|\mathcal{M}_{1}\right|+\left|\mathcal{M}_{3}\right|>\left|\mathcal{M}_{1}\right|+\left|\mathcal{M}_{2}\right|=\nu\left(G^{\prime \prime}\right) .
$$

Hence $\operatorname{reg} I\left(G^{\prime \prime}\right)=\nu\left(G^{\prime \prime}\right)+1 \leq \nu(G)$, and so we get the statement of the proposition.

Theorem 3.12. Let $G$ be a bicyclic graph with dumbbell $C_{n} \cdot P_{l} \cdot C_{m}$ such that $n \equiv$ $0,1(\bmod 3)$ and $m \equiv 2(\bmod 3)$. Then the following statements hold.

(i) $\nu(G)+1 \leq \operatorname{reg} I(G) \leq \nu(G)+2$; 
(ii) $\operatorname{reg} I(G)=\nu(G)+2$ if and only if $\nu(G)=\nu\left(G \backslash \Gamma_{G}\left(C_{m}\right)\right)$.

Proof. In Proposition 3.3 we proved $(i)$. In order to prove $(i i)$, we only need to show that $\nu\left(G \backslash \Gamma_{G}\left(C_{m}\right)\right)=\nu(G)$ implies $\operatorname{reg} I(G) \geq \nu(G)+2$, because the inverse implication follows from Proposition 3.11.

From Remark 3.8(i), $G \backslash \Gamma_{G}\left(C_{m}\right)=C_{m} \cup\left(\cup_{i=1}^{c} H_{i}\right)$ where each $H_{i}$ is either a forest or a unicyclic graph with cycle $C_{n}($ and $n \equiv 0,1(\bmod 3))$. Then, from Corollary 1.14, [2, Theorem 1.2] and Theorem 1.15 we get

$$
\begin{aligned}
\operatorname{reg} I\left(G \backslash \Gamma_{G}\left(C_{m}\right)\right) & =\operatorname{reg} I\left(C_{m}\right)+\operatorname{reg} I\left(\cup_{i=1}^{c} H_{i}\right)-1 \\
& =\left(\nu\left(C_{m}\right)+2\right)+\left(\nu\left(\cup_{i=1}^{c} H_{i}\right)+1\right)-1 \\
& =\nu\left(G \backslash \Gamma_{G}\left(C_{m}\right)\right)+2 \\
& =\nu(G)+2 .
\end{aligned}
$$

Finally, since $G \backslash \Gamma_{G}\left(C_{m}\right)$ is an induced subgraph of $G$ then we have $\operatorname{reg} I(G) \geq \nu(G)+2$.

3.2. Case II. The object of study of this subsection is the case where $n, m \equiv 2(\bmod 3)$, $l \geq 3$, and in particular when $\operatorname{reg} I(G)=\nu(G)+3$. More specifically, we shall give necessary and sufficient conditions for the equality $\operatorname{reg} I(G)=\nu(G)+3$.

Notation 3.13. Let $G$ be a bicyclic graph with dumbbell $C_{n} \cdot P_{l} \cdot C_{m}$ such that $n, m \equiv$ $2(\bmod 3)$ and $l \geq 3$. As in Notation 3.7, let $F_{1}, \ldots, F_{c}$ be the components of the graph $S_{G, 0}\left(C_{n}\right)$. We order the $F_{i}$ 's in such a way that $F_{1}$ is a unicyclic graph with cycle $C_{m}$, and for all $i>1$ we have that $F_{i}$ is a tree. The graph $S_{G, 2}\left(C_{n}\right)$ can be decomposed in components $H_{1}, \ldots, H_{c}$ where

$$
H_{i}=F_{i} \backslash\left\{v \in G \mid d\left(v, C_{n}\right) \leq 1\right\} .
$$

Remark 3.14. From the previous notation get the following simple remarks.

(i) The graph $G \backslash \Gamma_{G}\left(C_{n}\right)$ has a decomposition of the form

$$
G \backslash \Gamma_{G}\left(C_{n}\right)=C_{n} \bigcup\left(\bigcup_{i=1}^{c} H_{i}\right),
$$

and in particular

$$
\nu\left(G \backslash \Gamma_{G}\left(C_{n}\right)\right)=\nu\left(C_{n}\right)+\sum_{i=1}^{c} \nu\left(H_{i}\right)
$$

because $d\left(C_{n}, H_{i}\right) \geq 2$ for all $1 \leq i \leq c$ and $d\left(H_{i}, H_{j}\right) \geq 2$ for all $1 \leq i<j \leq c$.

(ii) Similarly, the graph $G \backslash \Gamma_{G}\left(C_{n} \cup C_{m}\right)$ has a decomposition of the form

$$
G \backslash \Gamma_{G}\left(C_{n} \cup C_{m}\right)=C_{n} \bigcup\left(\bigcup_{i=2}^{c} H_{i}\right) \bigcup\left(H_{1} \backslash \Gamma_{H_{1}}\left(C_{m}\right)\right),
$$

and in particular

$$
\nu\left(G \backslash \Gamma_{G}\left(C_{n} \cup C_{m}\right)\right)=\nu\left(C_{n}\right)+\sum_{i=2}^{c} \nu\left(H_{i}\right)+\nu\left(H_{1} \backslash \Gamma_{H_{1}}\left(C_{m}\right)\right) .
$$

(iii) For each $i=1, \ldots, c$, we have that $\left|F_{i} \cap C_{n}\right|=1$.

(iv) The statement of Lemma 3.10 also holds in this case, that is, if $\nu\left(H_{i}\right)=\nu\left(F_{i}\right)$ for all $1 \leq i \leq c$, then $\nu\left(G \backslash \Gamma_{G}\left(C_{n}\right)\right)=\nu(G)$.

(v) Due to the assumption $l \geq 3$, then we have that $C_{m}$ must be an induced subgraph of $H_{1}$. During this subsection and the next one we shall fundamentally use this fact, and it will allow us to inductively "separate" the two cycles $C_{n}$ and $C_{m}$. 
Lemma 3.15. Adopt Notation 3.13. If $\nu\left(H_{i}\right)=\nu\left(F_{i}\right)$ for all $1 \leq i \leq c$ and $\nu\left(H_{1}\right)=$ $\nu\left(H_{1} \backslash \Gamma_{H_{1}}\left(C_{m}\right)\right)$, then

$$
\nu\left(G \backslash \Gamma_{G}\left(C_{n} \cup C_{m}\right)\right)=\nu(G) .
$$

Proof. Since $G \backslash \Gamma_{G}\left(C_{n} \cup C_{m}\right)$ is an induced subgraph of $G$, then we have $\nu\left(G \backslash \Gamma_{G}\left(C_{n} \cup\right.\right.$ $\left.\left.C_{m}\right)\right) \leq \nu(G)$. From Remark 3.14(ii) we get

$$
\begin{aligned}
\nu\left(G \backslash \Gamma_{G}\left(C_{n} \cup C_{m}\right)\right) & =\nu\left(C_{n}\right)+\sum_{i=2}^{c} \nu\left(H_{i}\right)+\nu\left(H_{1} \backslash \Gamma_{H_{1}}\left(C_{m}\right)\right) \\
& =\nu\left(C_{n}\right)+\sum_{i=2}^{c} \nu\left(H_{i}\right)+\nu\left(H_{1}\right) \\
& =\nu\left(C_{n}\right)+\sum_{i=1}^{c} \nu\left(F_{i}\right) \\
& \geq \nu(G),
\end{aligned}
$$

and so $\nu\left(G \backslash \Gamma_{G}\left(C_{n} \cup C_{m}\right)\right)=\nu(G)$.

Proposition 3.16. Adopt Notation 3.13. If $\nu\left(G \backslash \Gamma_{G}\left(C_{n} \cup C_{m}\right)\right)<\nu(G)$, then

$$
\operatorname{reg} I(G) \leq \nu(G)+2 .
$$

Proof. From the contrapositive of Lemma 3.15, it follows that there exists some $i$ with $\nu\left(H_{i}\right)<\nu\left(F_{i}\right)$ or we have $\nu\left(H_{1} \backslash \Gamma_{H_{1}}\left(C_{m}\right)\right)<\nu\left(H_{1}\right)$. Then we divide the proof into two cases.

Case 1. In this case we assume that for some $1 \leq i \leq c$ we have $\nu\left(H_{i}\right)<\nu\left(F_{i}\right)$. This case follows similarly to Proposition 3.11. Let $x$ be the vertex in $F_{i} \cap C_{n}$, let us use the notations $G^{\prime}=G \backslash x$ and $G^{\prime \prime}=G \backslash N[x]$. Once more, we have the inequality

$$
\operatorname{reg} I(G) \leq \max \left\{\operatorname{reg} I\left(G^{\prime}\right), \operatorname{reg} I\left(G^{\prime \prime}\right)+1\right\} .
$$

Note that both $G^{\prime}$ and $G^{\prime \prime}$ are unicyclic graphs, and so we have reg $I\left(G^{\prime}\right) \leq \nu\left(G^{\prime}\right)+2$ and $\operatorname{reg} I\left(G^{\prime \prime}\right) \leq \nu\left(G^{\prime \prime}\right)+2$ (see Theorem 1.18). Since we have $\nu\left(G^{\prime}\right) \leq \nu(G)$ and $\nu\left(G^{\prime \prime}\right)+1 \leq$ $\nu(G)$ (see the proof of Proposition 3.11), then the inequality follows in this case.

Case 2. Now we suppose that $\nu\left(H_{1} \backslash \Gamma_{H_{1}}\left(C_{m}\right)\right)<\nu\left(H_{1}\right)$. Let $x$ be the vertex in $F_{1} \cap C_{n}$, let us use the notations $G^{\prime}=G \backslash x$ and $G^{\prime \prime}=G \backslash N[x]$. We use the inequality

$$
\operatorname{reg} I(G) \leq \max \left\{\operatorname{reg} I\left(G^{\prime}\right), \operatorname{reg} I\left(G^{\prime \prime}\right)+1\right\} .
$$

The graphs $G^{\prime}$ and $G^{\prime \prime}$ are unicyclic. For the graph $G^{\prime}$ we have $\operatorname{reg} I\left(G^{\prime}\right) \leq \nu\left(G^{\prime}\right)+2 \leq$ $\nu(G)+2$.

The graph $G^{\prime \prime}$ can be given as the disjoint union of $H_{1}$ and another graph $H$ defined by $H=G \backslash\left(F_{1} \cup N[x]\right)$, that is $G^{\prime \prime}=H \cup H_{1}$ and $d\left(H, H_{1}\right) \geq 2$. Thus it follows that $\nu\left(G^{\prime \prime}\right)=\nu(H)+\nu\left(H_{1}\right)$ and that $\operatorname{reg} I\left(G^{\prime \prime}\right)=\operatorname{reg} I(H)+\operatorname{reg} I\left(H_{1}\right)-1$ (see Corollary 1.14).

Since $H$ is a forest, Theorem 1.15 gives reg $I(H)=\nu(H)+1$. From [2, Corollary 3.11], it follows that $\operatorname{reg} I\left(H_{1}\right)=\nu\left(H_{1}\right)+1$. By summing up, we obtain that $\operatorname{reg} I\left(G^{\prime \prime}\right) \leq \nu\left(G^{\prime \prime}\right)+1$. So we get the inequality $\operatorname{reg} I\left(G^{\prime \prime}\right)+1 \leq \nu\left(G^{\prime \prime}\right)+2 \leq \nu(G)+2$, because $G^{\prime \prime}$ is an induced subgraph of $G$.

Now we are ready to completely describe the case where $\operatorname{reg} I(G)=\nu(G)+3$.

Theorem 3.17. Let $G$ be a bicyclic graph with dumbbell $C_{n} \cdot P_{l} \cdot C_{m}$. Then $\operatorname{reg} I(G)=$ $\nu(G)+3$ if and only if the following conditions are satisfied:

(i) $n \equiv 2(\bmod 3)$;

(ii) $m \equiv 2(\bmod 3)$; 
(iii) $l \geq 3$;

(iv) $\nu\left(G \backslash \Gamma_{G}\left(C_{n} \cup C_{m}\right)\right)=\nu(G)$.

Proof. In Proposition 3.3 we proved that the conditions (i), (ii) and (iii) are necessary, and from Proposition 3.16 we have that the condition $(i v)$ is also necessary. Hence, we only need to prove that $\operatorname{reg} I(G)=\nu(G)+3$ under these conditions.

Let $W=G \backslash \Gamma_{G}\left(C_{n} \cup C_{m}\right)$. From Remark 3.14(ii) and Corollary 1.14, obtain the equality

$$
\operatorname{reg}(I(W))=\operatorname{reg}\left(I\left(C_{n}\right)\right)+\operatorname{reg}\left(I\left(\cup_{i=2}^{c} H_{i}\right)\right)+\operatorname{reg}\left(I\left(H_{1} \backslash \Gamma_{H_{1}}\left(C_{m}\right)\right)\right)-2 .
$$

Note that the graph $H_{1} \backslash \Gamma_{H_{1}}\left(C_{m}\right)$ can be given as the disjoint union of the cycle $C_{m}$ and the forest $H=\left(H_{1} \backslash \Gamma_{H_{1}}\left(C_{m}\right)\right) \backslash C_{m}$, such that $d\left(H, C_{m}\right) \geq 2$. From Theorem 1.20 and Theorem 1.15 we get reg $I\left(C_{m}\right)=\nu\left(C_{m}\right)+2$ and reg $I(H)=\nu(H)+1$, respectively, and so Corollary 1.14 implies that $\operatorname{reg} I\left(H_{1} \backslash \Gamma_{H_{1}}\left(C_{m}\right)\right)=\operatorname{reg} I\left(C_{m}\right)+\operatorname{reg} I(H)-1=$ $\nu\left(C_{m}\right)+\nu(H)+2=\nu\left(H_{1} \backslash \Gamma_{H_{1}}\left(C_{m}\right)\right)+2$.

Therefore, by also using Theorem 1.20 and Theorem 1.15, we obtain

$$
\begin{aligned}
\operatorname{reg}(I(W)) & =\left(\nu\left(C_{n}\right)+2\right)+\left(\nu\left(\cup_{i=2}^{c} H_{i}\right)+1\right)+\left(\nu\left(H_{1} \backslash \Gamma_{H_{1}}\left(C_{m}\right)\right)+2\right)-2 \\
& =\nu(W)+3 \\
& =\nu(G)+3 .
\end{aligned}
$$

Since $W$ is an induced subgraph of $G$ then we get

$$
\operatorname{reg} I(G) \geq \operatorname{reg} I(W))=\nu(G)+3,
$$

and so from Theorem 1.18 the equality is obtained.

3.3. Case III. In this subsection we assume that $G$ is a bicyclic graph with dumbbell $C_{n} \cdot P_{l} \cdot C_{m}$ such that $n, m \equiv 2(\bmod 3)$ and $l \geq 3$. Now that we have characterized when $\operatorname{reg} I(G)=\nu(G)+3$, then we want to distinguish between $\operatorname{reg} I(G)=\nu(G)+1$ and $\operatorname{reg} I(G)=\nu(G)+2$.

Lemma 3.18. Adopt Notation 3.13. If $\nu(G)-\nu\left(G \backslash \Gamma_{G}\left(C_{n} \cup C_{m}\right)\right)=1$ then

$$
\operatorname{reg} I(G)=\nu(G)+2 .
$$

Proof. From Theorem 3.17 we have that $\operatorname{reg}(I(G)) \leq \nu(G)+2$. Using the same method as in Theorem 3.17, we can obtain a lower bound

$$
\operatorname{reg} I(G) \geq \operatorname{reg} I\left(G \backslash \Gamma_{G}\left(C_{n} \cup C_{m}\right)\right)=\nu\left(G \backslash \Gamma_{G}\left(C_{n} \cup C_{m}\right)\right)+3=\nu(G)+2,
$$

and so the equality follows.

Lemma 3.19. Adopt Notation 3.13. If $\nu(G)=\nu\left(G \backslash \Gamma_{G}\left(C_{n}\right)\right)$ then

$$
\operatorname{reg} I(G) \geq \nu(G)+2 \text {. }
$$

Symmetrically, the same argument holds for $C_{m}$.

Proof. The proof follows similarly to Theorem 3.12. From Remark 3.14(i), Corollary 1.14, Theorem 1.20 and Theorem 1.16 we get

$$
\begin{aligned}
\operatorname{reg} I\left(G \backslash \Gamma_{G}\left(C_{n}\right)\right) & =\operatorname{reg} I\left(C_{n}\right)+\operatorname{reg} I\left(\cup_{i=1}^{c} H_{i}\right)-1 \\
& \geq\left(\nu\left(C_{n}\right)+2\right)+\left(\nu\left(\cup_{i=1}^{c} H_{i}\right)+1\right)-1 \\
& \geq \nu\left(G \backslash \Gamma_{G}\left(C_{n}\right)\right)+2 \\
& \geq \nu(G)+2 .
\end{aligned}
$$

So the inequality follows from the fact that $G \backslash \Gamma_{G}\left(C_{n}\right)$ is an induced subgraph of $G$.

The following simple logical argument will be used several times in the next theorem. 
Observation 3.20. Let $P_{1}, P_{2}, P_{3}$ be boolean values, (i.e. true or false). Assume that $P_{1}$ is true if and only if $P_{2}$ and $P_{3}$ are true, that is

$$
P_{1} \Longleftrightarrow\left(P_{2} \wedge P_{3}\right) \text {. }
$$

Suppose that if $P_{2}$ is true then $P_{3}$ is false, that is

$$
P_{2} \Longrightarrow \neg P_{3} \text {. }
$$

Then, $P_{1}$ is false.

Notation 3.21. Let $X$ be a mathematical expression. Then, $P[X]$ represents a boolean value, which is true if $X$ is satisfied and false otherwise.

Taking into account the induced matching numbers $\nu(G), \nu\left(G \backslash \Gamma_{G}\left(C_{n} \cup C_{m}\right)\right), \nu(G \backslash$ $\left.\Gamma_{G}\left(C_{n}\right)\right)$ and $\nu\left(G \backslash \Gamma_{G}\left(C_{m}\right)\right)$, we can give necessary and sufficient conditions for the equality $\operatorname{reg} I(G)=\nu(G)+1$.

Theorem 3.22. Let $G$ be a bicyclic graph with dumbbell $C_{n} \cdot P_{l} \cdot C_{m}$ such that $n, m \equiv$ $2(\bmod 3)$ and $l \geq 3$. Then $\operatorname{reg} I(G)=\nu(G)+1$ if and only if the following conditions are satisfied:

(i) $\nu(G)-\nu\left(G \backslash \Gamma_{G}\left(C_{n} \cup C_{m}\right)\right)>1$;

(ii) $\nu(G)>\nu\left(G \backslash \Gamma_{G}\left(C_{n}\right)\right)$;

(iii) $\nu(G)>\nu\left(G \backslash \Gamma_{G}\left(C_{m}\right)\right)$.

Proof. From Theorem 3.17, Lemma 3.18 and Lemma 3.19, we have that the conditions $(i)$, (ii) and $($ iii $)$ are necessary. Hence, it is enough to prove $\operatorname{reg} I(G) \leq \nu(G)+1$ under these conditions.

For any $x \in G$ we denote $G^{\prime}=G \backslash x$ and $G^{\prime \prime}=G \backslash N[x]$. Then, we have the upper bound

$$
\operatorname{reg} I(G) \leq \max \left\{\operatorname{reg} I\left(G^{\prime}\right), \operatorname{reg} I\left(G^{\prime \prime}\right)+1\right\} .
$$

We shall prove that under the conditions $(i),(i i)$ and (iii) there exists a vertex $x \in C_{n}$ such that $\operatorname{reg} I\left(G^{\prime}\right) \leq \nu(G)+1$ and $\operatorname{reg} I\left(G^{\prime \prime}\right)+1 \leq \nu(G)+1$. We divide the proof into three steps.

Step 1. In this step we prove that for any $x \in C_{n}$ we have reg $I\left(G^{\prime}\right) \leq \nu(G)+1$. First we note the following two observations:

- It follows from Theorem 1.18 that $\operatorname{reg} I\left(G^{\prime}\right) \leq \nu\left(G^{\prime}\right)+2$. Hence, $\nu\left(G^{\prime}\right)<\nu(G)$ implies that $\operatorname{reg} I\left(G^{\prime}\right) \leq \nu\left(G^{\prime}\right)+2 \leq \nu(G)+1$.

- Since $G^{\prime}$ is a unicyclic graph, [2, Theorem 1.2] implies that $\operatorname{reg} I\left(G^{\prime}\right)=\nu\left(G^{\prime}\right)+2$ if and only if $\nu\left(G^{\prime}\right)=\nu\left(G^{\prime} \backslash \Gamma_{G^{\prime}}\left(C_{m}\right)\right)$.

Thus, it follows that

$$
\operatorname{reg} I\left(G^{\prime}\right)=\nu(G)+2 \Longleftrightarrow\left(\nu(G)=\nu\left(G^{\prime}\right) \text { and } \nu\left(G^{\prime}\right)=\nu\left(G^{\prime} \backslash \Gamma_{G^{\prime}}\left(C_{m}\right)\right)\right) .
$$

In Observation 3.20, let $P_{1}=P\left[\operatorname{reg} I\left(G^{\prime}\right)=\nu(G)+2\right], P_{2}=P\left[\nu(G)=\nu\left(G^{\prime}\right)\right]$ and $P_{3}=\left[\nu\left(G^{\prime}\right)=\nu\left(G^{\prime} \backslash \Gamma_{G^{\prime}}\left(C_{m}\right)\right)\right]$. From the logical argument of Observation 3.20, if we prove that $\nu\left(G^{\prime}\right)=\nu(G)$ implies $\nu\left(G^{\prime}\right)>\nu\left(G^{\prime} \backslash \Gamma_{G^{\prime}}\left(C_{m}\right)\right)$ then we get the desired inequality $\operatorname{reg} I\left(G^{\prime}\right) \leq \nu(G)+1$. Assume that $\nu(G)=\nu\left(G^{\prime}\right)$. From the hypothesis $\nu(G)>\nu\left(G \backslash \Gamma_{G}\left(C_{m}\right)\right)$ and the fact that $G^{\prime} \backslash \Gamma_{G^{\prime}}\left(C_{m}\right)$ is an induced subgraph of $G \backslash \Gamma_{G}\left(C_{m}\right)$, then we get

$$
\nu\left(G^{\prime}\right)=\nu(G)>\nu\left(G \backslash \Gamma_{G}\left(C_{m}\right)\right) \geq \nu\left(G^{\prime} \backslash \Gamma_{G^{\prime}}\left(C_{m}\right)\right) .
$$

Therefore, we have $\operatorname{reg} I\left(G^{\prime}\right) \leq \nu(G)+1$.

Step 2. Since $\nu(G)>\nu\left(G \backslash \Gamma_{G}\left(C_{n}\right)\right)$, it follows from Remark 3.14(iv) that there exists some $1 \leq i \leq c$ such that $\nu\left(F_{i}\right)>\nu\left(H_{i}\right)$. Following Notation 3.13, we have that $F_{1}$ is a 
unicyclic graph containing the cycle $C_{m}$ and that $F_{i}$ is a tree for all $i>1$. In this step, fix $i>1$ where $F_{i}$ is a tree and $\nu\left(F_{i}\right)>\nu\left(H_{i}\right)$.

Let $x$ be the vertex in $F_{i} \cap C_{n}$ and $H$ be the induced subgraph $H=G \backslash\left(F_{i} \cup N[x]\right)$. Note that $G^{\prime \prime}=H \cup H_{i}, d\left(H, H_{i}\right) \geq 2$ and $d\left(H, F_{i}\right) \geq 2$. Then

$$
\nu\left(G^{\prime \prime}\right)=\nu(H)+\nu\left(H_{i}\right)<\nu(H)+\nu\left(F_{i}\right) \leq \nu(G)
$$

follows from the condition $\nu\left(H_{i}\right)<\nu\left(F_{i}\right)$. So we have that $\nu\left(G^{\prime \prime}\right)<\nu(G)$.

As in Step 1, we note the following two observations:

- It follows from Theorem 1.18 that $\operatorname{reg} I\left(G^{\prime \prime}\right) \leq \nu\left(G^{\prime \prime}\right)+2$. Hence, $\nu\left(G^{\prime \prime}\right)+1<\nu(G)$ implies that $\operatorname{reg} I\left(G^{\prime \prime}\right)+1 \leq \nu\left(G^{\prime \prime}\right)+3 \leq \nu(G)+1$.

- Since $G^{\prime \prime}$ is a unicyclic graph, [2, Theorem 1.2] implies that reg $I\left(G^{\prime \prime}\right)=\nu\left(G^{\prime \prime}\right)+2$ if and only if $\nu\left(G^{\prime \prime}\right)=\nu\left(G^{\prime \prime} \backslash \Gamma_{G^{\prime \prime}}\left(C_{m}\right)\right)$.

So, we have that

$$
\operatorname{reg} I\left(G^{\prime \prime}\right)+1=\nu(G)+2 \Longleftrightarrow\left(\nu(G)=\nu\left(G^{\prime \prime}\right)+1 \text { and } \nu\left(G^{\prime \prime}\right)=\nu\left(G^{\prime \prime} \backslash \Gamma_{G^{\prime \prime}}\left(C_{m}\right)\right)\right) \text {. }
$$

Let $K$ be the induced subgraph defined by $K=\left(G \backslash \Gamma_{G}\left(C_{m}\right)\right) \backslash\left(F_{i} \cup N[x]\right)$. Since $i>1$ then $F_{i} \cap F_{1}=\emptyset$, and so we get the following statements:

- $G^{\prime \prime} \backslash \Gamma_{G^{\prime \prime}}\left(C_{m}\right)=K \cup H_{i}$, because $G^{\prime \prime}=H \cup H_{i}$ where $H=G \backslash\left(F_{i} \cup N[x]\right), C_{m} \subset H$ and $d\left(C_{m}, H_{i}\right) \geq 2$.

- $K \cup F_{i}$ is an induced subgraph of $G \backslash \Gamma_{G}\left(C_{m}\right)$.

- Since $d\left(K, F_{i}\right) \geq 2$ and $d\left(K, H_{i}\right) \geq 2$, we have the following inequalities

$$
\nu\left(G^{\prime \prime} \backslash \Gamma_{G^{\prime \prime}}\left(C_{m}\right)\right)=\nu(K)+\nu\left(H_{i}\right)<\nu(K)+\nu\left(F_{i}\right) \leq \nu\left(G \backslash \Gamma_{G}\left(C_{m}\right)\right) .
$$

In Observation 3.20, let $P_{1}=P\left[\operatorname{reg} I\left(G^{\prime \prime}\right)+1=\nu(G)+2\right], P_{2}=P\left[\nu(G)=\nu\left(G^{\prime \prime}\right)+1\right]$ and $P_{3}=\left[\nu\left(G^{\prime \prime}\right)=\nu\left(G^{\prime \prime} \backslash \Gamma_{G^{\prime}}\left(C_{m}\right)\right)\right]$. So it is enough to prove that $\nu(G)=\nu\left(G^{\prime \prime}\right)+1$ implies $\nu\left(G^{\prime \prime}\right)>\nu\left(G^{\prime \prime} \backslash \Gamma_{G^{\prime \prime}}\left(C_{m}\right)\right)$. Assuming $\nu(G)=\nu\left(G^{\prime \prime}\right)+1$ then we get

$$
\nu\left(G^{\prime \prime}\right)=\nu(G)-1>\nu\left(G \backslash \Gamma_{G}\left(C_{m}\right)\right)-1 \geq \nu\left(G^{\prime \prime} \backslash \Gamma_{G^{\prime \prime}}\left(C_{m}\right)\right) .
$$

Therefore, in this case we have $\operatorname{reg} I\left(G^{\prime \prime}\right)+1 \leq \nu(G)+1$.

Step 3. In this last step we assume that $\nu\left(F_{1}\right)>\nu\left(H_{1}\right)$ and that $\nu\left(F_{i}\right)=\nu\left(H_{i}\right)$ for all $i>1$. Let $x$ be the vertex in $F_{1} \cap C_{n}$, then as in Step 2 we have that:

- $\nu\left(G^{\prime \prime}\right)<\nu(G)$.

- $\operatorname{reg} I\left(G^{\prime \prime}\right)+1=\nu(G)+2 \Longleftrightarrow\left(\nu(G)=\nu\left(G^{\prime \prime}\right)+1\right.$ and $\left.\nu\left(G^{\prime \prime}\right)=\nu\left(G^{\prime \prime} \backslash \Gamma_{G^{\prime \prime}}\left(C_{m}\right)\right)\right)$. Once more, if we prove that $\nu(G)=\nu\left(G^{\prime \prime}\right)+1$ implies $\nu\left(G^{\prime \prime}\right)>\nu\left(G^{\prime \prime} \backslash \Gamma_{G^{\prime \prime}}\left(C_{m}\right)\right)$ then we obtain that $\operatorname{reg} I\left(G^{\prime \prime}\right)+1 \leq \nu(G)+1$.

We denote by $L$ the induced subgraph of $G^{\prime \prime} \backslash \Gamma_{G^{\prime \prime}}\left(C_{m}\right)$ given by

$$
L=\left(G^{\prime \prime} \backslash \Gamma_{G^{\prime \prime}}\left(C_{m}\right)\right) \backslash \Gamma_{G}\left(C_{n}\right) .
$$

Due to Remark 3.14(ii), the graph $L$ has the decomposition

$$
L=\left(C_{n} \backslash N_{C_{n}}[x]\right) \bigcup\left(\bigcup_{i=1}^{c} H_{i}\right) \bigcup\left(H_{1} \backslash \Gamma_{H_{1}}\left(C_{m}\right)\right),
$$

with all the disjoint components at distance at least two between each other, and so we have

$$
\nu(L)=\nu\left(C_{n} \backslash N_{C_{n}}[x]\right)+\sum_{i=2}^{c} \nu\left(H_{i}\right)+\nu\left(H_{1} \backslash \Gamma_{H_{1}}\left(C_{m}\right)\right) .
$$


By proceeding as in the proofs of Lemma 3.10 or Lemma 3.15, from the conditions $\nu\left(F_{i}\right)=\nu\left(H_{i}\right)$ for all $i>1$, we obtain

$$
\begin{aligned}
\nu(L) & =\nu\left(\left(C_{n} \backslash N_{C_{n}}[x]\right)\right)+\sum_{i=2}^{c} \nu\left(F_{i}\right)+\nu\left(H_{1} \backslash \Gamma_{H_{1}}\left(C_{m}\right)\right) \\
& \geq \nu\left(G^{\prime \prime} \backslash \Gamma_{G^{\prime \prime}}\left(C_{m}\right)\right) .
\end{aligned}
$$

Thus, $\nu(L)=\nu\left(G^{\prime \prime} \backslash \Gamma_{G^{\prime \prime}}\left(C_{m}\right)\right)$ because $L$ is an induced subgraph of $G^{\prime \prime} \backslash \Gamma_{G^{\prime \prime}}\left(C_{m}\right)$. We also have that $L$ is an induced subgraph of $G \backslash \Gamma_{G}\left(C_{n} \cup C_{m}\right)$ because we have the equality

$$
L=\left(G \backslash \Gamma_{G}\left(C_{n} \cup C_{m}\right)\right) \backslash N[x] .
$$

Finally, from the hypothesis $\nu(G)-\nu\left(G \backslash \Gamma_{G}\left(C_{n} \cup C_{m}\right)\right)>1$ we can obtain

$$
\nu\left(G^{\prime \prime}\right)=\nu(G)-1>\nu\left(G \backslash \Gamma_{G}\left(C_{n} \cup C_{m}\right)\right) \geq \nu(L)=\nu\left(G^{\prime \prime} \backslash \Gamma_{G^{\prime \prime}}\left(C_{m}\right)\right) .
$$

Therefore, in this case we also have $\operatorname{reg} I\left(G^{\prime \prime}\right)+1 \leq \nu(G)+1$.

So, we have finished the proof.

3.4. Case IV. In this short subsection we deal with the remaining case, we assume that $G$ is a bicyclic graph with dumbbell $C_{n} \cdot P_{l} \cdot C_{m}$ such that $n, m \equiv 2(\bmod 3)$ and $l \leq 2$.

When $l \leq 2$, the two cycles are too close to each other, and it is difficult to make a direct analysis (with our methods). Fortunately, using the complete characterization of the case $l \geq 3$, the problem can be solved with the Lozin transformation. Suppose that $x$ is a vertex on the bridge $P_{l}$ (at most two), then we apply the Lozin transformation of $G$ with respect to $x$, and obtain a bicyclic graph $\mathcal{L}_{x}(G)$ with dumbbell of the type $C_{n} \cdot P_{k} \cdot C_{m}$ where $k \geq 4$. From [25, Lemma 1] and [7, Theorem 1.1] we get the equality

$$
\operatorname{reg}\left(I\left(\mathcal{L}_{x}(G)\right)\right)-\nu\left(\mathcal{L}_{x}(G)\right)=\operatorname{reg}(I(G))-\nu(G) .
$$

Therefore we obtain a characterization in the following corollary.

Corollary 3.23. Let $G$ be a bicyclic graph with dumbbell $C_{n} \cdot P_{l} \cdot C_{m}$ such that $n, m \equiv$ $2(\bmod 3)$ and $l \leq 2$. Let $x$ be a point on the bridge $P_{l}$ and let $\mathcal{L}_{x}(G)$ be the Lozin transformation of $G$ with respect to $x$. Then we have that $\nu(G)+1 \leq \operatorname{reg} I(G) \leq \nu(G)+2$, and that $\operatorname{reg} I(G)=\nu(G)+1$ if and only if the following conditions are satisfied:

(i) $\nu\left(\mathcal{L}_{x}(G)\right)-\nu\left(\mathcal{L}_{x}(G) \backslash \Gamma_{\mathcal{L}_{x}(G)}\left(C_{n} \cup C_{m}\right)\right)>1$;

(ii) $\nu\left(\mathcal{L}_{x}(G)\right)>\nu\left(\mathcal{L}_{x}(G) \backslash \Gamma_{\mathcal{L}_{x}(G)}\left(C_{n}\right)\right)$;

(iii) $\nu\left(\mathcal{L}_{x}(G)\right)>\nu\left(\mathcal{L}_{x}(G) \backslash \Gamma_{\mathcal{L}_{x}(G)}\left(C_{m}\right)\right)$.

Proof. From Proposition 3.3, it follows that $\nu(G)+1 \leq \operatorname{reg} I(G) \leq \nu(G)+2$. Due to (4), we can apply the Lozin transformation and reduce the problem to the case where the bridge has more than three vertices. Finally, Theorem 3.22 gives us the result.

3.5. The characterization. Finally, the theorem below contains the characterization that we found.

Theorem 3.24. Let $G$ be a bicyclic graph with dumbbell $C_{n} \cdot P_{l} \cdot C_{m}$. The following statements hold.

(I) Let $n, m \equiv 0,1(\bmod 3)$, then $\operatorname{reg} I(G)=\nu(G)+1$.

(II) Let $n \equiv 0,1(\bmod 3)$ and $m \equiv 2(\bmod 3)$, then

$$
\nu(G)+1 \leq \operatorname{reg} I(G) \leq \nu(G)+2,
$$

and $\operatorname{reg} I(G)=\nu(G)+2$ if and only if $\nu(G)=\nu\left(G \backslash \Gamma_{G}\left(C_{m}\right)\right)$.

(III) Let $n, m \equiv 2(\bmod 3)$ and $l \geq 3$, then $\nu(G)+1 \leq \operatorname{reg} I(G) \leq \nu(G)+3$. Moreover:

(i) $\operatorname{reg} I(G)=\nu(G)+3$ if and only if $\nu\left(G \backslash \Gamma_{G}\left(C_{n} \cup C_{m}\right)\right)=\nu(G)$. 
(ii) $\operatorname{reg} I(G)=\nu(G)+1$ if and only if the following conditions hold:
(a) $\nu(G)-\nu\left(G \backslash \Gamma_{G}\left(C_{n} \cup C_{m}\right)\right)>1$;
(b) $\nu(G)>\nu\left(G \backslash \Gamma_{G}\left(C_{n}\right)\right)$;
(c) $\nu(G)>\nu\left(G \backslash \Gamma_{G}\left(C_{m}\right)\right)$.

(IV) Let $n, m \equiv 2(\bmod 3)$ and $l \leq 2$, then $\nu(G)+1 \leq \operatorname{reg} I(G) \leq \nu(G)+2$. If $x$ is an edge on $P_{l}$ and $\mathcal{L}_{x}(G)$ be the Lozin transformation of $G$ with respect to $x$, then $\operatorname{reg} I(G)=\nu(G)+1$ if and only if the following conditions are satisfied:
(a) $\nu\left(\mathcal{L}_{x}(G)\right)-\nu\left(\mathcal{L}_{x}(G) \backslash \Gamma_{\mathcal{L}_{x}(G)}\left(C_{n} \cup C_{m}\right)\right)>1$;
(b) $\nu\left(\mathcal{L}_{x}(G)\right)>\nu\left(\mathcal{L}_{x}(G) \backslash \Gamma_{\mathcal{L}_{x}(G)}\left(C_{n}\right)\right)$;
(c) $\nu\left(\mathcal{L}_{x}(G)\right)>\nu\left(\mathcal{L}_{x}(G) \backslash \Gamma_{\mathcal{L}_{x}(G)}\left(C_{m}\right)\right)$.

Proof. Statement (I) follows from Proposition 3.3. In Theorem 3.12, (II) is proved. By Theorem 3.17 and Theorem 3.22, we get (III). Finally, from Corollary 3.23, we obtain $(I V)$.

3.6. Examples. In this last subsection we give examples for each one of the statements in the characterization of Theorem 3.24.

Example 3.25. Statement (I) of Theorem 3.24. Let $G$ be the graph below.

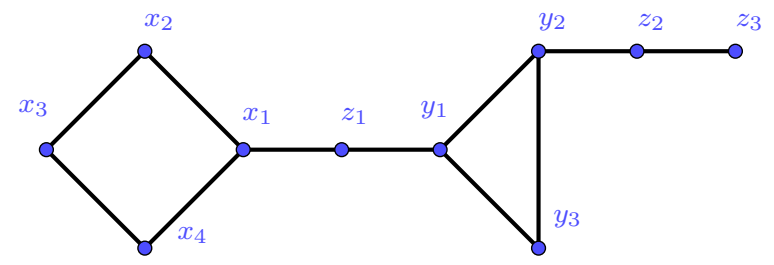

Then we have $\operatorname{reg} I(G)=4$ and $\nu(G)=3$.

Example 3.26. Statement (II) of Theorem 3.24. Let $G$ be the graph below.

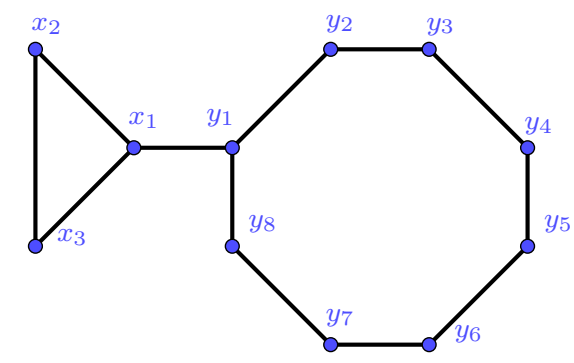

Then we have $\operatorname{reg} I(G)=5$ and $\nu(G)=3$.

If $G$ is the graph given below, then we have $\operatorname{reg} I(G)=5$ and $\nu(G)=4$.

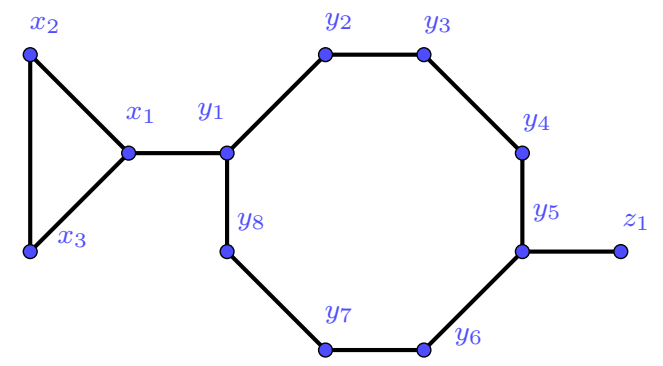

Example 3.27. Statement (III) of Theorem 3.24. 
Let $G$ be the graph given in Example 3.1. Then we have $\operatorname{reg} I(G)=6$ and $\nu(G)=3$. Let $G$ be the graph below.

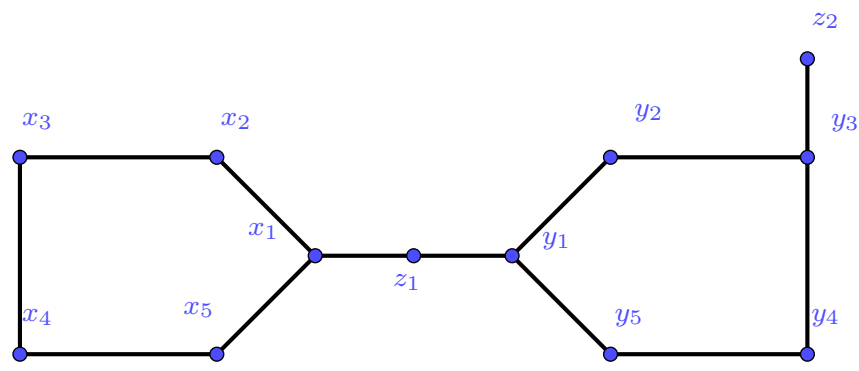

Then we have $\operatorname{reg} I(G)=5$ and $\nu(G)=3$.

Let $G$ be the graph given below and obtained by moving the whisker to the left.

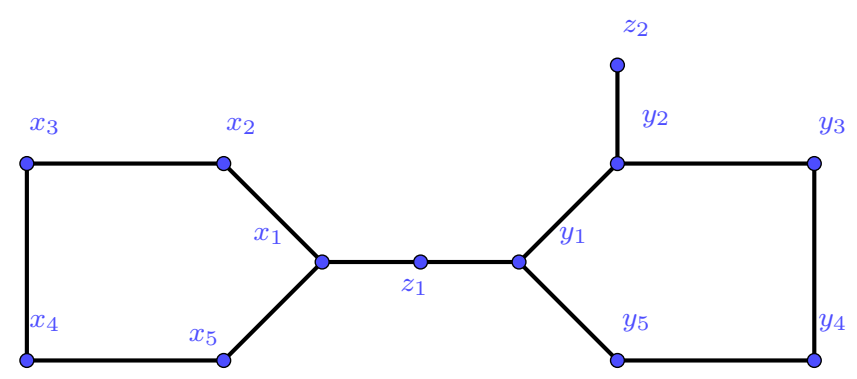

Then we have $\operatorname{reg} I(G)=5$ and $\nu(G)=4$.

Example 3.28. Statement (IV) of Theorem 3.24. Let $G$ be the graph below.

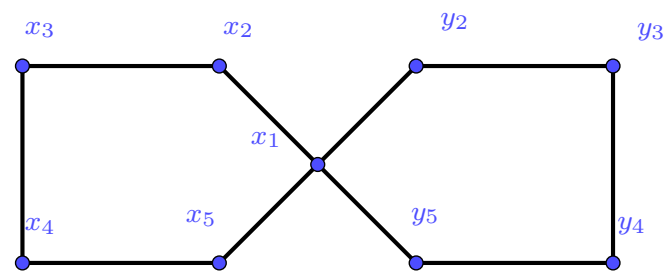

Then we have $\operatorname{reg} I(G)=4$ and $\nu(G)=2$.

Let $G$ be the graph given below and obtained by adding a whisker to the above graph at the join vertex $x_{1}$.

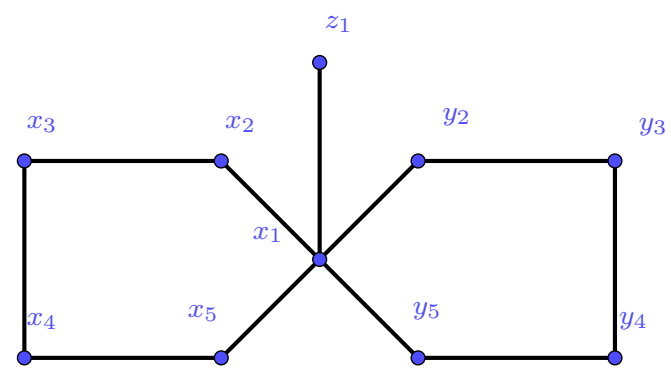

Then we have $\operatorname{reg} I(G)=4$ and $\nu(G)=3$. 


\section{Castelnuovo-Mumford Regularity of powers}

In this section, we study the regularity of the powers of $I\left(C_{n} \cdot P_{l} \cdot C_{m}\right)$ when $l \leq 2$. Our strategy to compute reg $I\left(C_{n} \cdot P_{l} \cdot C_{m}\right)^{q}$ for $q \geq 1$ relies on finding an upper bound and a lower bound on reg $I\left(C_{n} \cdot P_{l} \cdot C_{m}\right)^{q}$ where these bounds coincide and are equal to

$$
2 q+\operatorname{reg} I\left(C_{n} \cdot P_{l} \cdot C_{m}\right)-2 .
$$

In order to obtain an upper bound, we follow the even-connection argument given in [4, Theorem 5.2]. Then we proceed by looking at "nice" induced subgraphs of $C_{n} \cdot P_{l} \cdot C_{m}$ and we find a lower bound on $\operatorname{reg} I\left(C_{n} \cdot P_{l} \cdot C_{m}\right)^{q}$ which is equal to the found upper bound.

Let $I$ be an arbitrary ideal generated in degree $d$ and let $b_{q}:=\operatorname{reg}\left(I^{q}\right)-d q$ for $q \geq 1$. An interesting question is to study of the sequence $\left\{b_{i}\right\}_{i \geq 1}$. In [12] Eisenbud and Harris proved that if $\operatorname{dim}(R / I)=0$, then $\left\{b_{i}\right\}_{i \geq 1}$ is a weakly decreasing sequence of non-negative integers. In [5] Banerjee, Beyarslan and Hà conjectured that for any edge ideal, $\left\{b_{i}\right\}_{i \geq 1}$ is a weakly decreasing sequence (see [5, Conjecture 7.11]). For the edge ideal of any dumbbell graph with $l \leq 2$, we prove $b_{i}=b_{1}$ for all $i \geq 1$. However, we expect $b_{i} \leq b_{1}$ for all $i \geq 1$ for any graph.

Remark 4.1. From Theorem 2.4 and Theorem 2.16, for any $l \leq 2$ we have that

$$
\operatorname{reg} I\left(C_{n} \cdot P_{l} \cdot C_{m}\right) \geq\left\lfloor\frac{n+m+l+1}{3}\right\rfloor .
$$

The previous inequality is not satisfied when $l \geq 3$, because reg $I\left(C_{4} \cdot P_{3} \cdot C_{4}\right)=3$ and $\left\lfloor\frac{4+4+3+1}{3}\right\rfloor=4$.

As recalled earlier, we use the notation of even-connection from Banerjee [4, Theorem 5.2]. The following lemma is important in our treatment of the even-connected vertices, and its proof is similar to [4, Lemma 6.13].

Lemma 4.2. Let $G$ be a graph. As in Remark 1.10, let $G^{\prime}$ be the graph associated to $\left(I(G)^{q+1}: e_{1} \cdots e_{q}\right)^{\text {pol }}$. Suppose $u=p_{0}, p_{1}, \ldots, p_{2 s+1}=v$ is a path that even-connects $u$ and $v$ with respect to the $q$-fold $e_{1} \cdots e_{q}$. Then we have

$$
\bigcup_{i=0}^{2 s+1} N_{G^{\prime}}\left[p_{i}\right] \subset N_{G^{\prime}}[u] \cup N_{G^{\prime}}[v] .
$$

Proof. Let $U$ be the set of vertices $U=\left\{p_{0}, p_{1}, \ldots, p_{2 s+1}\right\}$. For each $1 \leq k \leq s$ we have that $p_{2 k-1} p_{2 k}=e_{j_{k}}$ for some $1 \leq j_{k} \leq q$, i.e. $u$ and $v$ are even connected with respect to the $s$-fold $e_{j_{1}} e_{j_{2}} \cdots e_{j_{s}}$.

Let $w$ be a vertex even-connected to some vertex $z \in U$ with respect to the $q$-fold $e_{1} \cdots e_{q}$. Then, there exists a path $z=r_{0}, r_{1}, \ldots, r_{2 t+1}=w$ that even-connects $z$ and $w$ with respect to the $q$-fold $e_{1} \cdots e_{q}$. Let $i$ be the largest integer such that $r_{i} \in U$. From the fact that $r_{0}=z \in U$, we have that the integer $i$ is well defined and $i \geq 0$. Let $k$ be an integer such that $p_{k}=r_{i}$.

The proof is now divided into four different cases depending on $i \bmod 2$ and $k \bmod 2$. When $i$ and $k$ are both odd integers, we have that $r_{i} r_{i+1}$ is equal to some edge of $\left\{e_{1}, e_{2}, \ldots, e_{q}\right\}$ and that $p_{k-1} p_{k}$ is not equal to any edge of $\left\{e_{j_{1}}, e_{j_{2}}, \ldots e_{j_{s}}\right\}$. By the definition of $i$ we have

$$
\left\{r_{i+1}, r_{i+2}, \ldots, r_{2 t+1}\right\} \cap U=\emptyset .
$$

So, in this case, it follows that

$$
u=p_{0}, \ldots, p_{k-1}, p_{k}=r_{i}, r_{i+1}, \ldots, r_{2 t+1}=w
$$

is a path that even-connects $u$ and $w$ with respect to the $q$-fold $e_{1} \cdots e_{q}$. 
The other three cases follow in a similar way. Therefore, we have that if $w$ even-connected to some $z \in U$, then $w$ is even-connected to either $u$ or $v$.

Now, we only need to prove that any $w \in N_{G}[z]$ for some $z \in U$ is even-connected to either $u$ or $v$. This part is simple, if $z=p_{2 j}$ then $u=p_{0}, \ldots, p_{2 j}=z, w$ is a path that even-connects $u$ and $w$, otherwise, if $z=p_{2 j-1}$ then $w, z=p_{2 j-1}, \ldots, p_{2 s+1}=v$ is a path that even-connects $w$ and $v$.

So we are done.

The next lemma is similar to [6, Lemma 5.1], but adapted to the current setting of a dumbbell.

Lemma 4.3. Let $G=C_{n} \cdot P_{l} \cdot C_{m}$. If $\left(I(G)^{q+1}: e_{1} \cdots e_{q}\right)$ is not a square-free monomial ideal and $G^{\prime}$ is the associated graph, then there exists a vertex $z$ which is even-connected to itself. Then, $G^{\prime}$ has a leaf and $N_{G^{\prime}}[z]$ contains one of the two cycles. In particular, if we denote the corresponding leaf by $e$, then $G_{e}^{\prime}$ is an induced subgraph of a unicyclic graph.

Proof. Suppose $z=p_{0}, p_{1}, \ldots, p_{2 l+1}=z$ is an even-connection of $z$ with itself. Let $0 \leq a<b \leq 2 l+1$ be integers such that $p_{a}, p_{a+1}, \ldots, p_{b}=p_{a}$ is an even-connection and $b-a$ is minimal. Then, $p_{a}, p_{a+1}, \ldots, p_{b}=p_{a}$ is a simple closed path lying on $C_{n} \cdot P_{l} \cdot C_{m}$ and so it is necessarily equal to either $C_{n}$ or $C_{m}$.

Finally, Lemma 4.2 implies that $N_{G^{\prime}}[z]$ contains either $C_{n}$ or $C_{m}$.

Lemma 4.4. Let $G=C_{n} \cdot P_{l} \cdot C_{m}$ with $l \leq 2$ and $H$ be a graph such that $G$ is a subgraph of $H$ with the same set of vertices (i.e., $V(H)=V(G)$ and $E(H) \supseteq E(G)$ ). For any two vertices $u, v \in H$ such that $\{u, v\} \notin E(G)$, we have that

$$
\operatorname{reg} I\left(H \backslash\left(N_{H}[u] \cup N_{H}[v]\right)\right) \leq \operatorname{reg} I(G)-1 .
$$

Proof. Let $K=N_{G}[u] \cap N_{G}[v]$. We divide the proof according to the cardinality $|K|$ of $K$. Notice that for the dumbbell $G$ we always have $0 \leq|K| \leq 2$.

Since $H \backslash\left(N_{H}[u] \cup N_{H}[v]\right)$ is an induced subgraph of $H \backslash\left(N_{G}[u] \cup N_{G}[v]\right)$, from Theorem 1.7(i), it is enough to prove that $\operatorname{reg} I\left(H \backslash\left(N_{G}[u] \cup N_{G}[v]\right)\right) \leq \operatorname{reg} I(G)-1$.

Step 1. Suppose that $|K|=0$. Then, the graph $H \backslash\left(N_{G}[u] \cup N_{G}[v]\right)$ is obtained by deleting at least 6 vertices, and so $\left|H \backslash\left(N_{G}[u] \cup N_{G}[v]\right)\right| \leq|G|-6 \leq n+m+l-8$. Note that we can add two vertices to $H \backslash\left(N_{G}[u] \cup N_{G}[v]\right)$ and connect them in such a way that we obtain a Hamiltonian path. Let $L$ be a graph obtained by adding two vertices and certain edges connecting these two new vertices, such that $L$ has a Hamiltonian path. Since $|L| \leq n+m+l-6$, Theorem 1.22 yields

$$
\operatorname{reg} I(L) \leq\left\lfloor\frac{n+m+l-5}{3}\right\rfloor+1=\left\lfloor\frac{n+m+l+1}{3}\right\rfloor-1,
$$

and by applying Remark 4.1, we get $\operatorname{reg} I(L) \leq \operatorname{reg} I(G)-1$. Since $H \backslash\left(N_{G}[u] \cup N_{G}[v]\right)$ is an induced subgraph of $L$, Theorem 1.7(i) implies that $\operatorname{reg} I\left(H \backslash\left(N_{G}[u] \cup N_{G}[v]\right)\right) \leq$ $\operatorname{reg} I(G)-1$.

Step 2. Suppose that $|K|=1$. Here the proof follows along the same lines of Step 1. In this case the graph $H \backslash\left(N_{G}[u] \cup N_{G}[v]\right)$ is obtained by deleting at least 5 vertices. Now, note that we can add one vertex to $H \backslash\left(N_{G}[u] \cup N_{G}[v]\right)$ and connect it in such a way that we obtain a Hamiltonian path. Let $L$ be a graph obtained by adding one vertex and certain edges connecting this new vertex, such that $L$ has a Hamiltonian path. Since $|L| \leq(n+m+l-2)-5+1=n+m+l-6$, then the rest of the proof follows as in Step 1.

Step 3. Suppose that $|K|=2$. In this case, note that one of the cycles is necessarily equal to $C_{4}$, say $C_{n}=C_{4}$, and that $u, v \in C_{4}$ with $\{u, v\} \notin E(G)$. Hence, it follows that $H \backslash\left(N_{G}[u] \cup N_{G}[v]\right)$ has a Hamiltonian path with $\leq m$ vertices if $l=2$ and $\leq m-1$ vertices 
if $l=1$. From Theorem 1.22 and Remark 4.1, then we have $\operatorname{reg} I\left(H \backslash\left(N_{G}[u] \cup N_{G}[v]\right)\right) \leq$ $\operatorname{reg} I(G)-1$.

So we are through.

Theorem 4.5. Let $G=C_{n} \cdot P_{l} \cdot C_{m}$ with $l \leq 2$ and $I=I(G)$ be its edge ideal, then

$$
\operatorname{reg}\left(I^{q+1}: e_{1} \cdots e_{q}\right) \leq \operatorname{reg} I
$$

for any $1 \leq q$ and any edges $e_{1}, \ldots, e_{q} \in E(G)$.

Proof. We split the proof into two cases.

Case 1. First, suppose $\left(I^{q+1}: e_{1} \cdots e_{q}\right)$ is a square-free monomial ideal. In this case $\left(I^{q+1}: e_{1} \cdots e_{q}\right)=I\left(G^{\prime}\right)$ where $G^{\prime}$ is a graph with $V(G)=V\left(G^{\prime}\right)$ and $E(G) \subseteq E\left(G^{\prime}\right)$. Let $E\left(G^{\prime}\right)=E(G) \cup\left\{a_{1}, \ldots, a_{r}\right\}$, then each edge $a_{i}$ is induced from even-connecting two different vertices (i.e., each $a_{i}$ is not a whisker). By Theorem 1.7, we have

$$
\operatorname{reg} I\left(G^{\prime}\right) \leq \max \left\{\operatorname{reg} I\left(G^{\prime} \backslash a_{1}\right), \operatorname{reg} I\left(G_{a_{1}}^{\prime}\right)+1\right\}
$$

Since $a_{1} \notin E(G)$, Lemma 4.4 implies that $\operatorname{reg} I\left(G_{a_{1}}^{\prime}\right)+1 \leq \operatorname{reg} I(G)$.

In the same way, for any subgraph $H=G^{\prime} \backslash\left\{a_{1}, \ldots, a_{i}\right\}$, since $V(H)=V(G)$ and $E(H) \supseteq E(G)$, Lemma 4.4 also gives us that

$$
\operatorname{reg}\left(I\left(H_{a_{i+1}}\right)\right)+1 \leq \operatorname{reg}(I(G)) .
$$

By continuing this process, we get $\operatorname{reg} I\left(G^{\prime}\right) \leq \operatorname{reg} I(G)$.

Case 2. Suppose $\left(I^{q+1}: e_{1} \cdots e_{q}\right)$ is not square-free and $G^{\prime}$ is the graph associated to $\left(I^{q+1}: e_{1} \cdots e_{q}\right)^{\mathrm{pol}}$. Let $\left\{b_{1}, b_{2}, \ldots, b_{s}\right\}$ be the subset of edges of $E\left(G^{\prime}\right) \backslash E(G)$ that are generated by square monomials (i.e., each $b_{i}$ is a whisker).

From Theorem 1.7 we have the inequality

$$
\operatorname{reg} I\left(G^{\prime}\right) \leq \max \left\{\operatorname{reg} I\left(G^{\prime} \backslash b_{1}\right), 1+\operatorname{reg} I\left(G_{b_{1}}^{\prime}\right)\right\} .
$$

Lemma 4.3 implies that one of the cycles is deleted from $G_{b_{1}}^{\prime}$, then there exists an edge $e \in G$ such that $d\left(e, G_{b_{1}}^{\prime}\right) \geq 2$. So, for such an edge $e$ we get that the disjoint union $G_{b_{1}}^{\prime} \cup e$ is an induced subgraph of $G^{\prime} \backslash b_{1}$. Thus, Theorem 1.7 and Corollary 1.14 yield that

$$
\operatorname{reg}\left(I\left(G_{b_{1}}^{\prime}\right)\right)+1=\operatorname{reg}\left(I\left(G_{b_{1}}^{\prime} \cup e\right)\right) \leq \operatorname{reg}\left(I\left(G^{\prime} \backslash b_{1}\right)\right) .
$$

Therefore, we obtain that $\operatorname{reg} I\left(G^{\prime}\right) \leq \operatorname{reg} I\left(G^{\prime} \backslash b_{1}\right)$.

By applying the same argument, it follows that

$$
\operatorname{reg} I\left(G^{\prime}\right) \leq \operatorname{reg} I\left(G^{\prime} \backslash b_{1}\right) \leq \operatorname{reg} I\left(G^{\prime} \backslash\left\{b_{1}, b_{2}\right\}\right) \leq \cdots \leq \operatorname{reg} I\left(G^{\prime} \backslash\left\{b_{1}, \ldots, b_{s}\right\}\right) .
$$

Since the graph $G^{\prime} \backslash\left\{b_{1}, \ldots, b_{s}\right\}$ has no whiskers, then Step 1 implies that

$$
\operatorname{reg} I\left(G^{\prime}\right) \leq \operatorname{reg} I\left(G^{\prime} \backslash\left\{b_{1}, \ldots, b_{s}\right\}\right) \leq \operatorname{reg} I(G) .
$$

Therefore, the proof is completed.

Remark 4.6. The previous theorem is a generalization of a work done by Gu in [15] for the case $l=1$.

Theorem 4.7. For the dumbbell graph $C_{n} \cdot P_{l} \cdot C_{m}$ with $l \leq 2$, we have

$$
\operatorname{reg} I\left(C_{n} \cdot P_{l} \cdot C_{m}\right)^{q} \geq 2 q+\operatorname{reg} I\left(C_{n} \cdot P_{l} \cdot C_{m}\right)-2,
$$

for any $q \geq 1$. 
Proof. Using the inequality reg $I\left(C_{n} \cdot P_{2} \cdot C_{m}\right)^{q} \geq 2 q+\nu\left(C_{n} \cdot P_{2} \cdot C_{m}\right)-1$ of Theorem 1.21, for the cases where $\operatorname{reg} I\left(C_{n} \cdot P_{l} \cdot C_{m}\right)=\nu\left(C_{n} \cdot P_{l} \cdot C_{m}\right)+1$ we get the expected inequality. We divide the proof in two halves, the cases $l=1$ and $l=2$.

Case 1 . Let $l=1$. We only need to focus on the case where $n, m \equiv 2(\bmod 3)$. Let $H$ be the induced subgraph of $C_{n} \cdot P_{1} \cdot C_{m}$ mentioned in the proof of Theorem 2.7, i.e. $H=\left(C_{n} \cdot P_{1} \cdot C_{m}\right) \backslash\left\{x_{n}\right\}=P_{n-1} \cdot C_{m}$. Using Theorem 2.4, Proposition 2.3 and the modularity $n, m \equiv 2(\bmod 3)$, we can check that

$$
\nu(H)=\nu\left(C_{n} \cdot P_{1} \cdot C_{m}\right)
$$

and that

$$
\nu(H)=\nu\left(H \backslash \Gamma_{H}\left(C_{m}\right)\right) .
$$

From Theorem 2.7 and [2, Theorem 1.2] we get

$$
\operatorname{reg} I\left(C_{n} \cdot P_{1} \cdot C_{m}\right)=\nu\left(C_{n} \cdot P_{1} \cdot C_{m}\right)+2=\nu(H)+2=\operatorname{reg} I(H) .
$$

Since $H$ is an induced subgraph of $C_{n} \cdot P_{1} \cdot C_{m}$, then from [2, Theorem 1.1] and [6, Corollay 4.3] we get the inequality

$\operatorname{reg} I\left(C_{n} \cdot P_{1} \cdot C_{m}\right)^{q} \geq \operatorname{reg} I(H)^{q}=2 q+\operatorname{reg} I(H)-2=2 q+\operatorname{reg} I\left(C_{n} \cdot P_{1} \cdot C_{m}\right)-2$.

Case 2 . Let $l=2$. We only need to focus on the cases where $n \equiv 0,1(\bmod 3)$ and $m \equiv 2(\bmod 3)$. We take the same induced subgraph $H$ as in Lemma 2.12. The induced subgraph $H=\left(C_{n} \cdot P_{2} \cdot C_{m}\right) \backslash\left\{x_{1}\right\}$ of $C_{n} \cdot P_{2} \cdot C_{m}$ is given as the union of a path of length $n-1$ and the cycle $C_{m}$, i.e., $H=P_{n-1} \cup C_{m}$.

By Theorem 2.13, for the cases $n \equiv 0,1(\bmod 3)$ and $m \equiv 2(\bmod 3)$, we have

$$
\operatorname{reg} I\left(C_{n} \cdot P_{2} \cdot C_{m}\right)=\nu\left(C_{n} \cdot P_{2} \cdot C_{m}\right)+2=\left\lfloor\frac{n}{3}\right\rfloor+\left\lfloor\frac{m}{3}\right\rfloor+2,
$$

and from Corollary 1.14, Theorem 1.15 and Theorem 1.20 we obtain

$$
\operatorname{reg} I(H)=\operatorname{reg}\left(I\left(P_{n-1}\right)\right)+\operatorname{reg}\left(I\left(C_{m}\right)\right)-1=\nu\left(P_{n-1}\right)+\nu\left(C_{m}\right)+2=\left\lfloor\frac{n}{3}\right\rfloor+\left\lfloor\frac{m}{3}\right\rfloor+2 .
$$

Hence, we get $\operatorname{reg} I\left(C_{n} \cdot P_{2} \cdot C_{m}\right)=\operatorname{reg} I(H)$. Finally, using [2, Theorem 1.1] and [6, Corollary 4.3], we get the inequality

$$
\operatorname{reg} I\left(C_{n} \cdot P_{2} \cdot C_{m}\right)^{q} \geq \operatorname{reg} I(H)^{q}=2 q+\operatorname{reg} I(H)-2=2 q+\operatorname{reg} I\left(C_{n} \cdot P_{2} \cdot C_{m}\right)-2 .
$$

Therefore, the proof is completed.

Theorem 4.8. For the dumbbell graph $C_{n} \cdot P_{l} \cdot C_{m}$ with $l \leq 2$, we have

$$
\operatorname{reg} I\left(C_{n} \cdot P_{l} \cdot C_{m}\right)^{q}=2 q+\operatorname{reg} I\left(C_{n} \cdot P_{l} \cdot C_{m}\right)-2
$$

for all $q \geq 1$.

Proof. It follows by Theorem 4.5, Theorem 1.11 and Theorem 4.7.

Remark 4.9. One may ask whether

$$
\operatorname{reg} I\left(C_{n} \cdot P_{l} \cdot C_{m}\right)^{q}=2 q+\operatorname{reg} I\left(C_{n} \cdot P_{l} \cdot C_{m}\right)-2
$$

always holds for given $n, m, l$ and $q$. Unfortunately, it is no longer true for any $n, m, l$ and $q$ as it can be seen from the following example:

$$
6=\operatorname{reg} I\left(C_{5} \cdot P_{3} \cdot C_{5}\right)^{2}<4+\operatorname{reg} I\left(C_{5} \cdot P_{3} \cdot C_{5}\right)-2=7 .
$$




\section{ACKNOWLEDGMENTS}

This project is originated from the summer school "Pragmatic 2017". The authors would like to sincerely express their gratitude to the organizers Alfio Ragusa, Elena Guardo, Francesco Russo, and Giuseppe Zappalà, and to the lecturers Brian Harbourne, Adam Van Tuyl, Enrico Carlini, and Tài Huy Hà. We are deeply grateful to the last lecturer for introducing this topic to us and for his mentoring. We are very grateful to the referee for valuable comments and suggestions that improved this paper in many ways. We thank Yan $\mathrm{Gu}$ for pointing out an error in the initial version. The computer algebra system Macaulay2 [14] was very helpful to compute several examples in the preparation of this paper.

\section{REFERENCES}

[1] A. Alilooee and A. Banerjee, Powers of edge ideals of regularity three bipartite graphs, J. Commut. Algebra 9 (2017), no. 4, 441-454. MR3713523

[2] A. Alilooee, S. Beyarslan, and S. Selvaraja, Regularity of powers of unicyclic graphs, Rocky Mountain J. Math. (2018). Advance publication.

[3] N. Altafi, N. Nemati, S. A. Seyed Fakhari, and S. Yassemi, Free resolution of powers of monomial ideals and Golod rings, Math. Scand. 120 (2017), no. 1, 59-67. MR3624006

[4] Arindam Banerjee, The regularity of powers of edge ideals, J. Algebraic Combin. 41 (2015), no. 2, 303-321.

[5] Arindam Banerjee, Selvi Beyarslan, and Huy Tai Ha, Regularity of edge ideals and their powers, arXiv preprint arXiv:1712.00887 (2017).

[6] Selvi Beyarslan, Huy Tài Hà, and Trân Nam Trung, Regularity of powers of forests and cycles, J. Algebraic Combin. 42 (2015), no. 4, 1077-1095.

[7] T. Biyikoglu and Y. Civan, Bounding Castelnuovo-Mumford regularity of graphs via Lozin's transformation, ArXiv e-prints (February 2013), available at 1302.3064.

[8] _ Castelnuovo-Mumford regularity of graphs, ArXiv e-prints (March 2015), available at 1503. 06018.

[9] Marc Chardin, Some results and questions on Castelnuovo-Mumford regularity, Syzygies and Hilbert functions, 2007, pp. 1-40.

[10] S. Dale Cutkosky, Jürgen Herzog, and Ngô Viêt Trung, Asymptotic behaviour of the CastelnuovoMumford regularity, Compositio Math. 118 (1999), no. 3, 243-261. MR1711319

[11] Hailong Dao, Craig Huneke, and Jay Schweig, Bounds on the regularity and projective dimension of ideals associated to graphs, J. Algebraic Combin. 38 (2013), no. 1, 37-55. MR3070118

[12] David Eisenbud and Joe Harris, Powers of ideals and fibers of morphisms, Math. Res. Lett. 17 (2010), no. 2, 267-273. MR2644374

[13] N. Erey, Powers of Edge Ideals with Linear Resolutions, ArXiv e-prints (March 2017), available at 1703.01561.

[14] Daniel R. Grayson and Michael E. Stillman, Macaulay2, a software system for research in algebraic geometry.

[15] Yan Gu, Regularity of powers of edge ideals of some graphs, Acta Math. Vietnam. 42 (2017), no. 3, 445-454. MR3667458

[16] Huy Tài Hà, Regularity of squarefree monomial ideals, Connections between algebra, combinatorics, and geometry, 2014, pp. 251-276. MR3213523

[17] Huy Tài Hà and Adam Van Tuyl, Monomial ideals, edge ideals of hypergraphs, and their graded Betti numbers, J. Algebraic Combin. 27 (2008), no. 2, 215-245. MR2375493

[18] Jürgen Herzog, A generalization of the Taylor complex construction, Comm. Algebra 35 (2007), no. 5, $1747-1756$.

[19] Jürgen Herzog and Takayuki Hibi, Monomial ideals, Graduate Texts in Mathematics, vol. 260, SpringerVerlag London, Ltd., London, 2011.

[20] Takayuki Hibi, Akihiro Higashitani, Kyouko Kimura, and Akiyoshi Tsuchiya, Dominating induced matchings of finite graphs and regularity of edge ideals, J. Algebraic Combin. 43 (2016), no. 1, 173-198. MR3439306

[21] A. Jayanthan and S Selvaraja, Asymptotic behavior of Castelnuovo-Mumford regularity of edge ideals of very well-covered graphs, ArXiv e-prints (August 2017), available at 1708.06883. 
[22] Gil Kalai and Roy Meshulam, Intersections of Leray complexes and regularity of monomial ideals, J. Combin. Theory Ser. A 113 (2006), no. 7, 1586-1592. MR2259083

[23] Mordechai Katzman, Characteristic-independence of Betti numbers of graph ideals, J. Combin. Theory Ser. A 113 (2006), no. 3, 435-454. MR2209703

[24] Vijay Kodiyalam, Asymptotic behaviour of Castelnuovo-Mumford regularity, Proc. Amer. Math. Soc. 128 (2000), no. 2, 407-411. MR1621961

[25] V. V. Lozin, On maximum induced matchings in bipartite graphs, Inform. Process. Lett. 81 (2002), no. 1, 7-11. MR1866807

[26] M. Moghimian, S. A. Seyed Fakhari, and S. Yassemi, Regularity of powers of edge ideal of whiskered cycles, Comm. Algebra 45 (2017), no. 3, 1246-1259. MR3573376

[27] P. Norouzi, S. A. Seyed Fakhari, and S. Yassemi, Regularity of Powers of edge ideal of very well-covered graphs, ArXiv e-prints (July 2017), available at 1707.04874.

[28] Ngô Viêt Trung and Hsin-Ju Wang, On the asymptotic linearity of Castelnuovo-Mumford regularity, J. Pure Appl. Algebra 201 (2005), no. 1-3, 42-48.

[29] Russ Woodroofe, Matchings, coverings, and Castelnuovo-Mumford regularity, J. Commut. Algebra 6 (2014), no. 2, 287-304. MR3249840

[30] Xinxian Zheng, Resolutions of facet ideals, Comm. Algebra 32 (2004), no. 6, 2301-2324.

Department de Matemàtiques i InformàticA, FACUltat de Matemàtiques i InformàticA,

Universitat de Barcelona, Gran Via de les Corts Catalanes, 585; 08007 Barcelona, Spain.

E-mail address: ycid@ub.edu

URL: http://www.ub.edu/arcades/ycid.html

Dipartimento di Matematica, Universit degli studi di Genova, Via Dodecaneso, 35, 16146 Genova GE, Italy

E-mail address: sepehr@dima.unige.it

Institut de mathématiques de Jussieu, Sorbonne Université, 4 Place Jussieu, 75005 Paris , FRANCE

E-mail address: navid.nemati@imj-prg.fr

Dipartimento di Matematica e Informatica, Viale A. Doria, 6 - 95100 - Catania, Italy

E-mail address: picone@dmi.unict.it 